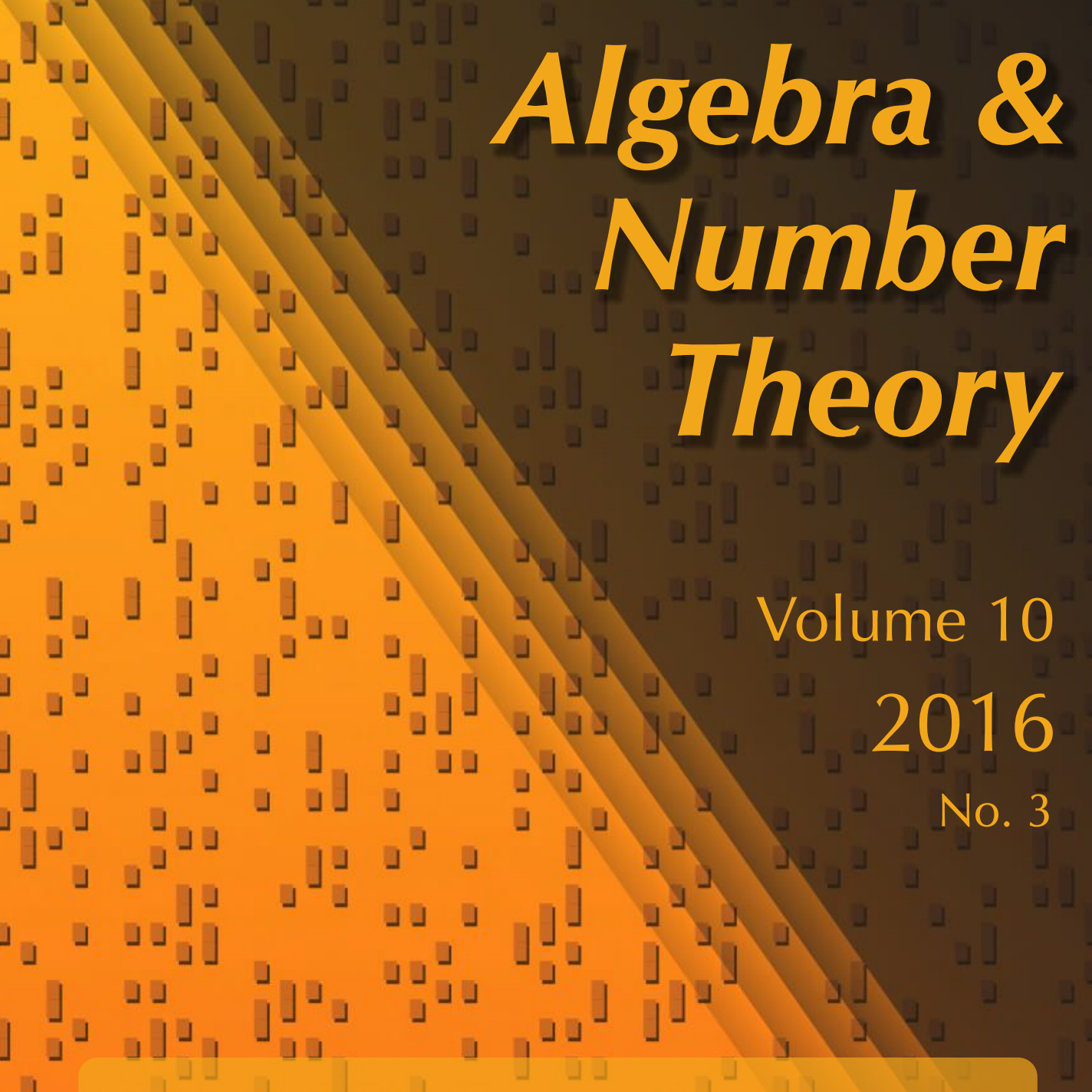




\title{
Discriminant formulas and applications
}

\author{
Kenneth Chan, Alexander A. Young and James J. Zhang
}

The discriminant is a classical invariant associated to algebras which are finite over their centers. It was shown recently by several authors that if the discriminant of $A$ is "sufficiently nontrivial" then it can be used to answer some difficult questions about $A$. Two such questions are: What is the automorphism group of $A$ ? Is $A$ Zariski cancellative?

We use the discriminant to study these questions for a class of (generalized) quantum Weyl algebras. Along the way, we give criteria for when such an algebra is finite over its center and prove two conjectures of Ceken, Wang, Palmieri and Zhang.

\section{Introduction}

In algebraic number theory, the discriminant takes on a familiar form: let $L$ be a Galois extension of the field $\mathbb{Q}$ and write $\mathcal{O}_{L}=\mathbb{Z}[\alpha] \cong \mathbb{Z}[x] /(f)$, where $f$ is the minimal polynomial (or the characteristic polynomial) of $\alpha$. Then we have

$$
\Delta_{L / \mathbb{Q}}=\prod_{i \neq j}\left(r_{i}-r_{j}\right)
$$

where $r_{1}, \ldots, r_{n}$ are the roots of $f$. In noncommutative algebra, the discriminant has long been used to study orders and lattices in a central simple algebra [Reiner 1975]. Recently, it has been shown that the discriminant plays a remarkable role in solving some classical and notoriously difficult questions:

(1) Automorphism problem: determining the full automorphism groups of noncommutative Artin-Schelter regular algebras [CPWZ 2015a; 2016].

(2) Zariski cancellation problem: concerning the cancellative property of noncommutative algebras such as skew polynomial rings [Bell and Zhang 2016].

(3) Isomorphism problem: finding a criterion for when two algebras are isomorphic, within certain classes of noncommutative algebras [CPWZ 2015b].

MSC2010: 16W20.

Keywords: discriminant, automorphism group, cancellation problem, quantum algebra, Clifford algebra, rings and algebras. 
Despite the usefulness of the discriminant in algebraic number theory, algebraic geometry and noncommutative algebra, it is extremely hard to compute, especially in high dimensional and high rank cases. In [CPWZ 2015a; 2016], the authors made two conjectures on discriminant formulas for some classes of noncommutative algebras. Our main aim is to prove these two conjectures.

Let $k$ be a base commutative domain and let $k^{\times}$be the set of invertible elements in $k$. The discriminant of a noncommutative algebra $A$ over a central subalgebra $Z \subseteq A$, denoted by $d(A / Z)$, will be reviewed in Section 1 . Let $q \in k^{\times}$be an invertible element in $k$ and let $A_{q}$ be the $q$-quantum Weyl algebra generated by $x$ and $y$ and subject to the relation $y x=q x y+1$. Our first result is:

Theorem 0.1. Let $q$ be a primitive $n$-th root of unity for some $n \geq 2$. Then the discriminant of $A_{q}$ over its center $Z\left(A_{q}\right)$ is

$$
d\left(A_{q} / Z\left(A_{q}\right)\right)=c(n m)^{n^{2}}\left((1-q)^{n} x^{n} y^{n}-1\right)^{n(n-1)},
$$

where $c$ is some element in $k^{\times}$and $m=\prod_{i=2}^{n-1}\left(1+q+\cdots+q^{i-1}\right)$. By convention, $m=1$ when $n=2$.

Theorem 0.1 answers [CPWZ 2016, Conjecture 5.3] affirmatively.

For $n \geq 2$, let $W_{n}$ be the $k$-algebra generated by $x_{1}, \ldots, x_{n}$ and subject to the relations $x_{i} x_{j}+x_{j} x_{i}=1$ for all $i \neq j$ [CPWZ 2015a, Introduction]. This algebra is called a (-1)-quantum Weyl algebra [CPWZ 2015b, Introduction]. Let

$$
M:=\left(\begin{array}{cccc}
2 x_{1}^{2} & 1 & \cdots & 1 \\
1 & 2 x_{2}^{2} & \cdots & 1 \\
\vdots & \vdots & \ddots & \vdots \\
1 & 1 & \cdots & 2 x_{n}^{2}
\end{array}\right) .
$$

Let $Z$ denote the central subalgebra $k\left[x_{1}^{2}, \ldots, x_{n}^{2}\right] \subseteq W_{n}$. Our second result is:

Theorem 0.2. Suppose 2 is invertible in $k$. Then the discriminant of $W_{n}$ over the subalgebra $Z$ is

$$
d\left(W_{n} / Z\right)=c(\operatorname{det} M)^{2^{n-1}},
$$

where $c$ is an element in $k^{\times}$.

Theorem 0.2 answers [CPWZ 2015a, Question 4.12(2)] affirmatively.

These results suggest that the discriminant has elegant expressions in some situations. Because of its usefulness, more discriminant formulas should be established; see Lemma 6.4.

This paper contains other related results which we now describe. Let $T$ be a commutative algebra over $k$ and let $\boldsymbol{q}:=\left\{q_{i j} \in T^{\times} \mid 1 \leq i<j \leq n\right\}$ and 
$\mathcal{A}:=\left\{a_{i j} \in T \mid 1 \leq i<j \leq n\right\}$ be sets of elements in $T$. The skew polynomial ring $T_{q}\left[x_{1}, \ldots, x_{n}\right]$ is a $T$-algebra generated by $x_{1}, \ldots, x_{n}$ and subject to the relations

$$
x_{j} x_{i}=q_{i j} x_{i} x_{j} \quad \text { for all } 1 \leq i<j \leq n .
$$

A generalized quantum Weyl algebra associated to $(\boldsymbol{q}, \mathcal{A})$ is a $T$-central filtered algebra of the form

$$
V_{n}(\boldsymbol{q}, \mathcal{A})=\frac{T\left\langle x_{1}, \ldots, x_{n}\right\rangle}{\left(x_{j} x_{i}-q_{i j} x_{i} x_{j}-a_{i j} \mid i<j\right)}
$$

such that the associated graded ring $\operatorname{gr} V_{n}(\boldsymbol{q}, \mathcal{A})$ is naturally isomorphic to the skew polynomial ring $T_{\boldsymbol{q}}\left[x_{1}, \ldots, x_{n}\right]$. Another way of constructing $V_{n}(\boldsymbol{q}, \mathcal{A})$ is to use an iterated Ore extension starting with $T$. To calculate the discriminant of $V_{n}(\boldsymbol{q}, \mathcal{A})$ over its center, one needs to determine the center of $V_{n}(\boldsymbol{q}, \mathcal{A})$. The discriminant is defined whenever $V_{n}(\boldsymbol{q}, \mathcal{A})$ is a finite module over a central subring $Z$ [CPWZ 2016], and it is most useful when $V_{n}(\boldsymbol{q}, \mathcal{A})$ is a free module over $Z$ [CPWZ 2015a]. Since $\operatorname{gr} V_{n}(\boldsymbol{q}, \mathcal{A})$ is isomorphic to $T_{\boldsymbol{q}}\left[x_{1}, \ldots, x_{n}\right]$, it is a finite module over its center if and only if each $q_{i j}$ is a root of unity. Using this, we can show that the algebra $V_{n}(\boldsymbol{q}, \mathcal{A})$ is a finite module over its center if and only if the parameters $q_{i j}$ are all nontrivial roots of unity. Also, when the center of $V_{n}(\boldsymbol{q}, \mathcal{A})$ is a polynomial ring, $V_{n}(\boldsymbol{q}, \mathcal{A})$ is a finitely generated free module over its center. The following useful result concerns the centers of $V_{n}(\boldsymbol{q}, \mathcal{A})$ and $T_{\boldsymbol{q}}\left[x_{1}, \ldots, x_{n}\right]$.

To state it, we need some notation. When $q_{i j}$ is a root of unity, there are two integers $k_{i j}$ and $d_{i j}$ such that

$$
q_{i j}=\exp \left(2 \pi \sqrt{-1} k_{i j} / d_{i j}\right),
$$

where $d_{i j}:=o\left(q_{i j}\right)<\infty,\left|k_{i j}\right|<d_{i j}$ and $\left(k_{i j}, d_{i j}\right)=1$. Further, we can choose $k_{i j}$ so that $k_{i j}=-k_{j i}$, since $q_{j i}=q_{i j}^{-1}$. Let $L_{i}=\operatorname{lcm}\left\{d_{i j} \mid j=1, \ldots, n\right\}$. Let $\bar{Y}$ be the $n \times n$ matrix $\left(k_{i j} L_{i} / d_{i j}\right)_{n \times n}$. For each prime $p$, define $\bar{Y}_{p}=\bar{Y} \otimes \mathbb{F}_{p}$. Let $m$ be any natural number. Let $I_{p, m}$ be the set containing $i$ such that $L_{i} \in p^{m} \mathbb{Z}-p^{m+1} \mathbb{Z}$. Finally, let $\bar{Y}_{p, m}$ be the submatrix of $\bar{Y}_{p}$ taken from the rows and columns with indices $i \in I_{p, m}$.

Theorem 0.3. Suppose $q_{i j}$ is a root of unity and not 1 for all $i<j$.

(1) The center of $T_{q}\left[x_{1}, \ldots, x_{n}\right]$ is a polynomial ring if and only if it is of the form $T\left[x_{1}^{L_{1}}, \ldots, x_{n}^{L_{n}}\right]$, if and only if $\operatorname{det}\left(\bar{Y}_{p, m}\right) \neq 0$ in $\mathbb{F}_{p}$ for all primes $p$ and all integers $m>0$ such that $I_{p, m} \neq \varnothing$.

(2) If the center of $T_{q}\left[x_{1}, \ldots, x_{n}\right]$ is the subalgebra $T\left[x_{1}^{L_{1}}, \ldots, x_{n}^{L_{n}}\right]$, then the center of $V_{n}(\boldsymbol{q}, \mathcal{A})$ is the same subalgebra and $V_{n}(\boldsymbol{q}, \mathcal{A})$ is finitely generated and free over it. 
The above criterion can be simplified when $n=3$ or 4 [Corollaries 5.4 and 5.5]. The point of Theorem 0.3 is that it provides an explicit linear algebra criterion for when the center of $T_{q}\left[x_{1}, \ldots, x_{n}\right]$ is isomorphic to a polynomial ring.

Question 0.4. Suppose that $A:=V_{n}(\boldsymbol{q}, \mathcal{A})$ is finitely generated and free over its center $Z$. What is the discriminant $d(A / Z)$ ?

Theorems 0.1 and 0.2 answer this question for two special cases.

A secondary goal of this paper is to provide some quick applications. These discriminant formulas have potential applications in algebraic geometry, number theory and the study of Clifford algebras. In Section 8 (the final section), we give some immediate applications of discriminants to the cancellation problem and the automorphism problem for several classes of noncommutative algebras.

Let us briefly review some definitions. An algebra $A$ is called cancellative if $A[t] \cong B[t]$ for some algebra $B$ implies $A \cong B$. Let $\operatorname{Aut}(A)$ be the group of all algebra automorphisms of $A$. Let $A$ be connected graded. An algebra automorphism $g$ of $A$ is called unipotent if

$$
g(v)=v+(\text { higher degree terms })
$$

for all homogeneous elements $v \in A$. Let $\operatorname{Aut}_{\text {uni }}(A)$ denote the subgroup of $\operatorname{Aut}(A)$ consisting of all unipotent automorphisms [CPWZ 2016, after Theorem 3.1]. When $\operatorname{Aut}_{\text {uni }}(A)$ is trivial, $\operatorname{Aut}(A)$ is usually small and easy to handle. We will give a criterion on when $\operatorname{Aut}_{\text {uni }}(A)$ is trivial.

Let $A$ be a domain and let $F$ be a subset of $A$. Let $\operatorname{Sw}(F)$ be the set of $g \in A$ such that $f=a g b$ for some $a, b \in A$ and $0 \neq f \in F$. Let $D_{1}(F)$ be the $k$-subalgebra of $A$ generated by $\operatorname{Sw}(F)$. For $n>2$, we define $D_{n}(F)=D_{1}\left(D_{n-1}(F)\right)$ inductively, and define $D(F)=\bigcup_{n \geq 1} D_{n}(F)$. This algebra is called the $F$-divisor subalgebra of $A$. When $F=\{d(A / Z)\}, D(F)$ is called the discriminant-divisor subalgebra of $A$ and is denoted by $\mathbb{D}(A)$. The main result in Section 8 is the following.

Theorem 0.5. Suppose $k$ is a field of characteristic zero. Let A be a connected graded domain of finite Gelfand-Kirillov dimension. Assume that $A$ is finitely generated and free over its center. If $\mathbb{D}(A)=A$, then $A$ is cancellative and $\operatorname{Aut}_{\text {uni }}(A)=\{1\}$.

The above theorem can be applied to some Artin-Schelter regular algebras of global dimension 4 in Examples 6.3 and 8.4. Further applications are certainly expected.

This paper is organized as follows. Background material about discriminants is provided in Section 1. We prove Theorem 0.1 in Section 2 and Theorem 0.2 in Section 3. Sections 4-6 concern the question of when $T_{\boldsymbol{q}}\left[x_{1}, \ldots, x_{n}\right]$ and $V_{n}(\boldsymbol{q}, \mathcal{A})$ are finitely generated and free over their centers and contain the proof of Theorem 0.3. In Section 7, we review and introduce some invariants related to discriminants, 
locally nilpotent derivations, and automorphisms, which will be used in Section 8 . In Section 8, some applications are provided and Theorem 0.5 is proven.

\section{Preliminaries}

In this section we recall some definitions and basic properties of the discriminant. A basic reference is [CPWZ 2015a, Section 1].

Throughout, let $k$ be a base commutative domain and let everything be over $k$. Let $A$ be an algebra and let $Z$ be a central subalgebra of $A$ such that $A$ is finitely generated and free over $Z$. A modified version of the discriminant was introduced in [CPWZ 2016] when $A$ is not free over $Z$; however, in this paper, we only consider the case when $A$ is finitely generated and free over $Z$. Let $r$ be the rank of $A$ over $Z$.

We embed $A$ in the endomorphism ring $\operatorname{End}\left(A_{Z}\right)$ by sending $a \in A$ to the left multiplication $l_{a}: A \rightarrow A$. Since $A$ is free over $Z$ of rank $r, \operatorname{End}\left(A_{Z}\right) \cong M_{r \times r}(Z)$. Define the trace function

$$
\operatorname{tr}: A \longrightarrow \operatorname{End}\left(A_{Z}\right) \cong M_{r \times r}(Z) \stackrel{\operatorname{tr}_{m}}{\longrightarrow} Z,
$$

where $\operatorname{tr}_{m}$ is the usual matrix trace. The trace function $\operatorname{tr}$ is independent of the choice of basis of $A$ over $Z$.

Definition 1.1. [CPWZ 2015a, Definition 1.3(3)] Retain the above notation. Suppose that $A$ is a free module over a central subalgebra $Z$ with a $Z$-basis $\left\{z_{1}, \ldots, z_{r}\right\}$. The discriminant of $A$ over $Z$ is

$$
d(A / Z)=\operatorname{det}\left(\operatorname{tr}\left(z_{i} z_{j}\right)\right)_{r \times r} \in Z .
$$

By [CPWZ 2015a, Proposition 1.4(2)], $d(A / Z)$ is unique up to a scalar in $Z^{\times}$. For $x, y \in Z$, we use the notation $x={ }_{Z^{\times}} y$ to indicate that $x=c y$ for some $c \in Z^{\times}$. So $d(A / Z)=_{Z^{\times}} \operatorname{det}\left(\operatorname{tr}\left(z_{i} z_{j}\right)\right)_{r \times r}$ as in [CPWZ 2015a, Definition 1.3(3)]. The following lemma is easy.

Lemma 1.2. Retain the notation of Definition 1.1. Let $\left(A^{\prime}, Z^{\prime}\right)$ be another pair of algebras such that $Z^{\prime}$ is a central subalgebra of $A^{\prime}$ and $A^{\prime}$ is a free $Z^{\prime}$-module of rank $r$. Let $g: A \rightarrow A^{\prime}$ be an algebra homomorphism such that:

(a) $g(Z) \subseteq Z^{\prime}$.

(b) $\left\{g\left(z_{1}\right), \ldots, g\left(z_{r}\right)\right\}$ is a $Z^{\prime}$-basis of $A^{\prime}$.

Then $g(d(A / Z))={ }_{\left(Z^{\prime}\right)} \times d\left(A^{\prime} / Z^{\prime}\right)$.

Proof. For any $a \in A$, we define $a^{\prime}=g(a)$. Write $a z_{i}=\sum_{j=1}^{r} a_{i j} z_{j}$ for all $i$. By applying $g$ to the last equation, we have $a^{\prime} z_{i}^{\prime}=\sum_{j=1}^{r} a_{i j}^{\prime} z_{j}^{\prime}$. By definition (E1.0.1), $\operatorname{tr}(a)=\sum_{i} a_{i i}$ and

$$
\operatorname{tr}(g(a))=\operatorname{tr}\left(a^{\prime}\right)=\sum_{i} a_{i i}^{\prime}=g\left(\sum_{i} a_{i i}\right)=g(\operatorname{tr}(a))
$$


for all $a \in A$. By Definition 1.1 and the above equation,

$$
g(d(A / Z))=g\left(\operatorname{det}\left(\operatorname{tr}\left(z_{i} z_{j}\right)\right)_{r \times r}\right)=\operatorname{det}\left(\operatorname{tr}\left(z_{i}^{\prime} z_{j}^{\prime}\right)\right)_{r \times r}={ }_{\left(Z^{\prime}\right)^{\prime}} d\left(A^{\prime} / Z^{\prime}\right) .
$$

Let $Z$ be a central subalgebra of $A$ and consider an Ore set $C \subset Z$. Then the localization $Z C^{-1}$ is central in $A C^{-1}$.

Lemma 1.3. Let $Z$ be a central subalgebra of $A$. Suppose $A$ is free over $Z$ of rank $r$. Then $A C^{-1}$ is free over $Z C^{-1}$ of rank $r$. As a consequence,

$$
d\left(A C^{-1} / Z C^{-1}\right)={ }_{\left(Z C^{-1}\right) \times} d(A / Z) .
$$

Proof. Let $\left\{z_{1}, \ldots, z_{r}\right\}$ be a $Z$-basis of $A$. Then it is also a $Z C^{-1}$-basis of $A C^{-1}$. The consequence follows from Lemma 1.2.

We will need the following result from [CPWZ 2016]. We use $T$ in place of $k$ to denote a commutative domain.

Proposition 1.4. Let $T$ be a commutative domain and let $A=T_{q}\left[x_{1}, \ldots, x_{n}\right]$. Suppose $Z:=T\left[x_{1}^{\alpha_{1}}, \ldots, x_{n}^{\alpha_{n}}\right]$ is a central subalgebra of $A$, where the $\alpha_{i}$ are positive integers.

(1) [CPWZ 2016, Proposition 2.8] Let $r=\prod_{i=1}^{n} \alpha_{i}$. Then

$$
d(A / Z)={ }_{T^{\times}} r^{r}\left(\prod_{i=1}^{n} x_{i}^{\alpha_{i}-1}\right)^{r} .
$$

(2) If $n=2, Z=T\left[x_{1}^{m}, x_{2}^{m}\right]$, and $q_{12}$ is a primitive $m$-th root of unity, then

$$
d(A / Z)={ }_{T \times} m^{2 m^{2}}\left(x_{1}^{m} x_{2}^{m}\right)^{m(m-1)} .
$$

(3) If $q_{i j}=-1$ for all $i<j$ and $\alpha_{i}=2$ for all $i$, then

$$
d(A / Z)=T_{T^{\times}} 2^{n 2^{n}}\left(\prod_{i=1}^{n} x_{i}^{2}\right)^{2^{n-1}} .
$$

Proof. Parts (2) and (3) are special cases of part (1).

The next lemma is a special case of [CPWZ 2016, Proposition 4.10]. Suppose $Z$ is a central subalgebra of $A$ and $A$ is free over $Z$ of rank $r<\infty$. We fix a $Z$-basis of $A$, say $b:=\left\{b_{1}=1, b_{2}, \ldots, b_{r}\right\}$. Suppose $A$ is an $\mathbb{N}$-filtered algebra such that the associated graded ring gr $A$ is a domain. For any element $f \in A$, let gr $f$ denote the associated element in $\operatorname{gr} A$. Let $\operatorname{gr} b$ denote the set $\left\{\operatorname{gr} b_{1}, \ldots, \operatorname{gr} b_{r}\right\}$, which is a subset of $\operatorname{gr} A$.

Lemma 1.5. Retain the above notation. Suppose that $\operatorname{gr} A$ is finitely generated and free over gr $Z$ with basis gr $b$. Then

$$
\operatorname{gr}(d(A / Z))={ }_{(\operatorname{gr} Z)} \times d(\operatorname{gr} A / \operatorname{gr} Z) .
$$




\section{Discriminant of $A_{q}$ over its center}

Let $T$ be a commutative domain and let $q \in T^{\times}$be a primitive $n$-th root of unity for some $n \geq 2$. Let $A_{q}$ be the $q$-quantum Weyl algebra over $T$ generated by $x$ and $y$ and subject to the relation $y x=q x y+a$ for some $a \in T$. This agrees with the definition of $A_{q}$ given in the Introduction when $T=k$ and $a=1$. It is easy to check that the center of $A_{q}$, denoted by $Z\left(A_{q}\right)$, is $T\left[x^{n}, y^{n}\right]$, and that $A_{q}$ is free over $Z\left(A_{q}\right)$ of rank $n^{2}$. A $Z\left(A_{q}\right)$-basis of $A_{q}$ is $\mathcal{B}:=\left\{x^{i} y^{j} \mid 0 \leq i, j \leq n-1\right\}$. The aim of this section is to compute the discriminant $d\left(A_{q} / Z\left(A_{q}\right)\right)$.

Let $A^{\prime}$ be the $T$-subalgebra of $A_{q}$ generated by $x^{\prime}:=(1-q) x$ and $y$. Since $y x^{\prime}=q x^{\prime} y+(1-q) a$ and $(1-q)$ may not be invertible, there is no obvious algebra homomorphism from $A_{q}$ to $A^{\prime}$. Let $Z^{\prime}$ be the subalgebra $T\left[\left(x^{\prime}\right)^{n}, y^{n}\right]$ which is the center of $A^{\prime}$.

Lemma 2.1. Retain the above notation. Then

$$
d\left(A^{\prime} / Z^{\prime}\right)=(1-q)^{n^{2}(n-1)} d\left(A_{q} / Z\left(A_{q}\right)\right) .
$$

Proof. Let $\operatorname{tr}^{\prime}: A^{\prime} \rightarrow Z^{\prime}$ be the trace function defined in (E1.0.1). We use this trace function to compute the discriminant $d\left(A^{\prime} / Z^{\prime}\right)$.

Let $\mathcal{B}^{\prime}:=\left\{\left(x^{\prime}\right)^{i} y^{j}\right\}_{0 \leq i, j \leq n-1}$. Then $\mathcal{B}^{\prime}$ is a $Z^{\prime}$-basis of $A^{\prime}$. Note that $A^{\prime}$ and $A_{q}$ have the same ring of fractions and $Z\left(A_{q}\right)$ and $Z^{\prime}$ have the same fraction field. Since the trace function is independent of the choice of basis, we have $\operatorname{tr}^{\prime}(a)=\operatorname{tr}(a)$ for all $a \in A^{\prime}$.

Picking any two elements $b_{s}=x^{i_{s}} y^{j_{s}}$ and $b_{t}=x^{i_{t}} y^{j_{t}}$ in $\mathcal{B}$, we have corresponding elements $b_{s}^{\prime}=\left(x^{\prime}\right)^{i_{s}} y^{j_{s}}$ and $b_{t}^{\prime}=\left(x^{\prime}\right)^{i_{t}} y^{j_{t}}$ in $\mathcal{B}$. Hence

$$
\operatorname{tr}^{\prime}\left(b_{s}^{\prime} b_{t}^{\prime}\right)=\operatorname{tr}\left((1-q)^{i_{s}+i_{t}} b_{s} b_{t}\right)=(1-q)^{i_{s}+i_{t}} \operatorname{tr}\left(b_{s} b_{t}\right) .
$$

By definition, $d\left(A^{\prime} / Z^{\prime}\right)=\operatorname{det}\left[\operatorname{tr}^{\prime}\left(b_{s}^{\prime} b_{t}^{\prime}\right)_{b_{s}^{\prime}, b_{t}^{\prime} \in \mathcal{B}^{\prime}}\right]$. Hence we have

$$
\begin{aligned}
d\left(A^{\prime} / Z^{\prime}\right) & =\operatorname{det}\left[\left(\operatorname{tr}^{\prime}\left(b_{s}^{\prime} b_{t}^{\prime}\right)\right)_{b_{s}^{\prime}, b_{t}^{\prime} \in \mathcal{B}^{\prime}}\right]=\operatorname{det}\left[\left((1-q)^{i_{s}+i_{t}} \operatorname{tr}\left(b_{s} b_{t}\right)\right)_{b_{s}, b_{t} \in \mathcal{B}}\right] \\
& =(1-q)^{N} \operatorname{det}\left[\left(\operatorname{tr}\left(b_{s} b_{t}\right)\right)_{b_{s}, b_{t} \in \mathcal{B}}\right]=(1-q)^{N} d\left(A_{q} / Z\left(A_{q}\right)\right),
\end{aligned}
$$

where

$$
N=\sum_{\text {all } i_{s}, i_{t}}\left(i_{s}+i_{t}\right)=2 \sum_{\text {all } i_{s}} i_{s}=2 n(0+1+2+\cdots+(n-1))=n^{2}(n-1) .
$$

The assertion follows.

Following the above lemma, we first compute $d\left(A^{\prime} / Z^{\prime}\right)$. We can rewrite $A^{\prime}$ as $T\left\langle x^{\prime}, y\right\rangle /\left(y x^{\prime}-q x^{\prime} y-(1-q) a\right)$ so that the positions of $x^{\prime}$ and $y$ are more symmetrical. 
Let $C=\left\{\left(y^{n}\right)^{i} \mid i \geq 1\right\}$. Consider the localizations $Z^{\prime \prime}:=Z^{\prime} C^{-1}$ and $A^{\prime \prime}:=A^{\prime} C^{-1}$. Let

$$
x^{\prime \prime}:=x^{\prime}-a y^{-1}=(1-q) x-\left(a y^{-n}\right) y^{n-1} \in A^{\prime \prime} .
$$

Lemma 2.2. Retain the above notation. The following hold:

(1) $y x^{\prime \prime}-q x^{\prime \prime} y=0$.

(2) $A^{\prime \prime}:=A^{\prime} C^{-1}$ is generated by $T,\left(y^{n}\right)^{-1}, x^{\prime \prime}$ and $y$.

(3) $\left(x^{\prime \prime}\right)^{n}$ is central and $d\left(A^{\prime \prime} / Z^{\prime \prime}\right)={ }_{\left(Z^{\prime \prime}\right) \times} n^{2 n^{2}}\left(\left(x^{\prime \prime}\right)^{n} y^{n}\right)^{n(n-1)}$.

(4) $d\left(A^{\prime \prime} / Z^{\prime \prime}\right)={ }_{\left(Z^{\prime \prime}\right)} \times n^{2 n^{2}}\left((1-q)^{n} x^{n} y^{n}-a^{n}\right)^{n(n-1)}$.

Proof. (1) We have $y x^{\prime \prime}-q x^{\prime \prime} y=y\left((1-q) x-a y^{-1}\right)-q\left((1-q) x-a y^{-1}\right) y=0$.

(2) This is clear.

(3) Since $q^{n}=1,\left(x^{\prime \prime}\right)^{n}$ commutes with $y$ by part (1). By part (2), $\left(x^{\prime \prime}\right)^{n}$ commutes with every element in $A^{\prime \prime}$.

Consider an algebra homomorphism $g: T_{q}\left[x_{1}, x_{2}\right] \rightarrow A^{\prime \prime}$ determined by $g\left(x_{1}\right)=x^{\prime \prime}$ and $g\left(x_{2}\right)=y$. Then the center of $B:=T_{q}\left[x_{1}, x_{2}\right]$ is $R:=T\left[x_{1}^{n}, x_{2}^{n}\right]$ and $\left\{x_{1}^{i} x_{2}^{j} \mid\right.$ $0 \leq i, j \leq n-1\}$ is an $R$-basis of $B$. It is clear that $A^{\prime \prime}$ is free of rank $n^{2}$ and that $A^{\prime \prime}=\sum_{0 \leq i, j \leq n-1}\left(x^{\prime}\right)^{i} y^{j} Z^{\prime \prime}$. Hence $\left\{\left(x^{\prime \prime}\right)^{i} y^{j} \mid 0 \leq i, j \leq n-1\right\}$ is a $Z^{\prime \prime}$-basis of $A^{\prime \prime}$. Then the hypotheses of Lemma 1.2 hold. Applying Lemma 1.2 to $g$, we have $g(d(B / R))={ }_{\left(Z^{\prime}\right) \times} d\left(A^{\prime \prime} / Z^{\prime \prime}\right)$. By Proposition 1.4(2), $d(B / R)=n^{2 n^{2}}\left(x_{1}^{n} x_{2}^{n}\right)^{n(n-1)}$. Therefore, $d\left(A^{\prime \prime} / Z^{\prime \prime}\right)={ }_{\left(Z^{\prime}\right)} \times n^{2 n^{2}}\left(\left(x^{\prime \prime}\right)^{n} y^{n}\right)^{n(n-1)}$.

(4) In the following, we will let $\psi=y^{-1}, z=x^{\prime \prime}$ and $p=q^{-1}$. The commutation relation between $x^{\prime}$ and $\psi$ is

$$
\psi x^{\prime}=(1-q) \psi x=(1-q)\left(p x \psi-p a \psi^{2}\right)=p x^{\prime} \psi-(p-1) a \psi^{2} .
$$

Recall that $z=x^{\prime \prime}=x^{\prime}-a \psi$. Write $z^{n}=\sum_{i=0}^{n} c_{i}\left(x^{\prime}\right)^{i} \psi^{n-i}$. Since $z^{n}$ is central (see part (3)), we have $c_{i}=0$ unless $i=0, n$. It is clear that $c_{n}=1$. Next we determine $c_{0}$. Since $A^{\prime \prime}$ is a free module over $Z^{\prime \prime}$ with basis $\left\{\left(x^{\prime}\right)^{i} \psi^{j} \mid 0 \leq i, j \leq n-1\right\}$, we can work modulo the right $Z^{\prime \prime}$-submodule $W$ generated by $\left(x^{\prime}\right)^{i} \psi^{j}$, where $0<i<n$ and $0 \leq j<n$. Let $\equiv$ denote equivalence $\bmod W$.

By induction, for $i=1, \ldots, n-1$, we have

$$
\psi^{i} x^{\prime}=p^{i} x^{\prime} \psi^{i}-\left(p^{i}-1\right)\left(a \psi^{i+1}\right) .
$$

Then $\psi^{i} x^{\prime} \equiv-\left(p^{i}-1\right)\left(a \psi^{i+1}\right)$. For each $1 \leq j \leq n-1$, write

$$
z^{j}=\sum_{i=0}^{j} c_{i}^{j}\left(x^{\prime}\right)^{i} \psi^{j-i} .
$$


Then $x^{\prime} z^{j} \in W$ for all $j<n-1$ and $x^{\prime} z^{n-1} \equiv\left(x^{\prime}\right)^{n}$. For each $j$, we have $\psi^{j-1} z^{n-j}=$ $\sum_{i=0}^{n-j} d_{i}^{j}\left(x^{\prime}\right)^{i} \psi^{n-1-i}$ for some $d_{i}^{j} \in Z^{\prime}$, so

$$
x^{\prime} \psi^{j-1} z^{n-j} \in W
$$

for all $j \geq 2$. By the above computation and (E2.2.1)-(E2.2.3), we have

$$
\begin{aligned}
z^{n}-\left(x^{\prime}\right)^{n} & =\left(x^{\prime}-a \psi\right) z^{n-1}-\left(x^{\prime}\right)^{n} \\
& =x^{\prime} z^{n-1}-\left(x^{\prime}\right)^{n}-a \psi z^{n-1} \\
& \equiv-a \psi\left(x^{\prime}-a \psi\right) z^{n-2} \\
& \equiv-a\left(p x^{\prime} \psi-(p-1) a \psi^{2}-a \psi^{2}\right) z^{n-2} \\
& \equiv-a(-p a) \psi^{2} z^{n-2}-a p x^{\prime} \psi z^{n-2} \\
& \equiv-a(-p a) \psi^{2} z^{n-2} \\
& \equiv-a(-p a)\left(\psi^{2} x-a \psi^{3}\right) z^{n-3} \\
& \equiv-a(-p a)\left(-p^{2} a\right) \psi^{3} z^{n-3} \\
& \vdots \\
& \equiv-a(-p a)\left(-p^{2} a\right) \cdots\left(-p^{n-1} a\right) \psi^{n} \\
& =(-a)^{n} p^{(n-1) n / 2} \psi^{n}=-a^{n} \psi^{n} .
\end{aligned}
$$

Therefore,

$$
z^{n} \equiv-a^{n} \psi^{n}+\left(x^{\prime}\right)^{n} .
$$

Hence $c_{0}=-a^{n}$ and $z^{n}=\left(x^{\prime}\right)^{n}-a^{n} \psi^{n}$. Combining all of the above, we have

$$
\left(x^{\prime \prime}\right)^{n} y^{n}=\left(\left(x^{\prime}\right)^{n}-a^{n} \psi^{n}\right) y^{n}=\left(x^{\prime}\right)^{n} y^{n}-a^{n}=(1-q)^{n} x^{n} y^{n}-a^{n} .
$$

Part (4) follows from part (3) and the above formula.

Lemma 2.3. The discriminant of $A^{\prime}$ over its center $Z^{\prime}$ is

$$
d\left(A^{\prime} / Z^{\prime}\right)={ }_{T \times} n^{2 n^{2}}\left((1-q)^{n} x^{n} y^{n}-a^{n}\right)^{n(n-1)} .
$$

Proof. Let $g$ be the embedding of $A^{\prime}$ into $A^{\prime \prime}=A^{\prime} C^{-1}$, viewed as an inclusion. By Lemma $1.2, g$ sends $d\left(A^{\prime} / Z^{\prime}\right)$ to $d\left(A^{\prime \prime} / Z^{\prime \prime}\right)$. Combining this fact with Lemma 2.2(4), we have

$$
\begin{aligned}
d\left(A^{\prime} / Z^{\prime}\right) & ={ }_{\left(Z^{\prime \prime}\right)} \times g\left(d\left(A^{\prime} / Z\left(A^{\prime}\right)\right)\right)==_{\left(Z^{\prime \prime}\right)} \times d\left(A^{\prime \prime} / Z^{\prime \prime}\right) \\
& ={ }_{\left(Z^{\prime \prime}\right)} \times n^{2 n^{2}}\left((1-q)^{n} x^{n} y^{n}-a^{n}\right)^{n(n-1)} .
\end{aligned}
$$

Let $\Phi$ be the element $d\left(A^{\prime} / Z^{\prime}\right)\left\{n^{2 n^{2}}\left((1-q)^{n} x^{n} y^{n}-a^{n}\right)^{n(n-1)}\right\}^{-1}$, which can be viewed as an element in the quotient ring of $A^{\prime}$. By the above equation, $\Phi$ is in $\left(Z^{\prime \prime}\right)^{\times}$. Since $Z^{\prime \prime}=T\left[\left(x^{\prime}\right)^{n}, y^{ \pm n}\right], \Phi$ is of the form $\alpha y^{s n}$ for some $\alpha \in T^{\times}$ 
and some $s$. By symmetry, $\Phi$ is also of the form $\beta\left(x^{\prime}\right)^{t n}$ for some $\beta \in T^{\times}$and some $t$. Hence $s=t=0, \alpha=\beta \in T^{\times}$and $\Phi=\alpha \in T^{\times}$. Therefore, $d\left(A^{\prime} / Z^{\prime}\right)=$ $\alpha n^{2 n^{2}}\left((1-q)^{n} x^{n} y^{n}-a^{n}\right)^{n(n-1)}$ and the assertion follows.

Now let

$$
m:=\prod_{i=2}^{n-1}\left(1+q+\cdots+q^{i-1}\right) .
$$

We can show that $n=(1-q)^{n-1} m$ by first factoring the polynomial $x^{n}-1 \in T[x]$ and dividing by $(x-1)$ :

$$
x^{n}-1=\prod_{i=0}^{n-1}\left(x-q^{i}\right) \quad \Longrightarrow \quad \sum_{i=0}^{n-1} x^{i}=\frac{x^{n}-1}{x-1}=\prod_{i=1}^{n-1}\left(x-q^{i}\right) .
$$

We then substitute 1 for $x$ as follows:

$$
n=\prod_{i=1}^{n-1}\left(1-q^{i}\right)=(1-q)^{n-1} \prod_{i=2}^{n-1}\left(1+q+\cdots+q^{i-1}\right)=(1-q)^{n-1} m .
$$

Now we are ready to prove the main result of this section, which also recovers Theorem 0.1.

Theorem 2.4. Retain the above notation. The discriminant of $A_{q}$ over its center $Z\left(A_{q}\right)$ is

$$
d\left(A_{q} / Z\left(A_{q}\right)\right)=_{T^{\times}}(n m)^{n^{2}}\left((1-q)^{n} x^{n} y^{n}-a^{n}\right)^{n(n-1)} .
$$

Proof. Using Lemmas 2.1 and 2.3 and equation (E2.3.2), we have

$$
(1-q)^{n^{2}(n-1)} d\left(A_{q} / Z\left(A_{q}\right)\right)={ }_{T^{\times}}\left(n m(1-q)^{n-1}\right)^{n^{2}}\left((1-q)^{n} x^{n} y^{n}-a^{n}\right)^{n(n-1)} .
$$

Since $A_{q}$ is a domain, we obtain

$$
d\left(A_{q} / Z\left(A_{q}\right)\right)={ }_{T^{\times}}(n m)^{n^{2}}\left((1-q)^{n} x^{n} y^{n}-a^{n}\right)^{n(n-1)} .
$$

Remark 2.5. (1) By [CPWZ 2016, Lemma 2.7(7)], the integer $n$ in Theorem 2.4 is nonzero in $T$. However, $n$ and $m$ may not be invertible in general.

(2) Theorem 0.1 is clearly a consequence of Theorem 2.4.

A slight generalization of Theorem 2.4 is the following.

Theorem 2.6. Let $T$ be a commutative domain and $q \in T^{\times}$be a primitive $n$-th root of unity. Let $B$ be the T-algebra of the form

$$
\frac{T\langle x, y\rangle}{\left(y x-q x y=a, x^{n}=b, y^{n}=c\right)},
$$


where $a, b, c \in T$. Suppose that $B$ is a free module over $T$ with basis $\left\{x^{i} y^{j} \mid\right.$ $0 \leq i, j \leq n-1\}$. Then $d(B / T)={ }_{T^{\times}}(n m)^{n^{2}}\left((1-q)^{n} x^{n} y^{n}-a^{n}\right)^{n(n-1)}$, where $m$ is given in (E2.3.1).

Proof. First note that it is well-known and easy to check that $T$ is the center of $B$.

Recall that $A_{q}$ is the algebra of the form $T\langle x, y\rangle /(y x-q x y=a)$. There is a natural algebra homomorphism $g$ from $A_{q}$ to $B$ sending $x$ to $x$ and $y$ to $y$ and $t \in T$ to $t \in T$. Then the hypotheses in Lemma 1.2 hold. By Lemma 1.2, $g\left(d\left(A_{q} / Z\left(A_{q}\right)\right)\right)=d(B / T)$. Now the assertion follows from Theorem 2.4.

\section{Discriminant of Clifford algebras}

In this section we assume that $2^{-1} \in k$. We fix an integer $n \geq 2$.

Let $T$ be a commutative domain and let $\mathcal{A}:=\left\{a_{i j} \mid 1 \leq i<j \leq n\right\}$ be a set of scalars in $T$. We write $a_{j i}=a_{i j}$ if $i<j$. Let $V_{n}(\mathcal{A})$ be the $T$-algebra generated by $x_{1}, \ldots, x_{n}$ and subject to the relations

$$
x_{i} x_{j}+x_{j} x_{i}=a_{i j} \quad \text { for all } i \neq j .
$$

This algebra was studied in [CPWZ 2015a; 2015b]. Some basic properties of $V_{n}(\mathcal{A})$ are given in [CPWZ 2015a, Section 4]. Let $M_{1}$ be the matrix

$$
M_{1}:=\left(\begin{array}{cccc}
2 x_{1}^{2} & a_{12} & \cdots & a_{1 n} \\
a_{21} & 2 x_{2}^{2} & \cdots & a_{2 n} \\
\vdots & \vdots & \ddots & \vdots \\
a_{n 1} & a_{n 2} & \cdots & 2 x_{n}^{2}
\end{array}\right) .
$$

This is a symmetric matrix with entries in $Z:=T\left[x_{1}^{2}, \ldots, x_{n}^{2}\right]$. We will define a sequence of matrices $M_{i}$ later. Note that $Z$ is a central subalgebra of $V_{n}(\mathcal{A})$. If we write $M_{1}=\left(m_{i j, 1}\right)_{n \times n}$, then $m_{i j, 1}=x_{j} x_{i}+x_{i} x_{j}$ for all $i, j$.

The algebra $V_{n}(\mathcal{A})$ is a Clifford algebra over $Z$. We will recall the definition of the Clifford algebra associated to a quadratic form in the second half of this section. In the next few lemmas, we are basically diagonalizing the quadratic form, which is elementary and well-known in the classical case; see [Lam 2005, Chapter I, Corollary 2.4] for some related material. Since we need an explicit construction to complete the proof of our main result, details will be provided below.

We will introduce a sequence of new variables starting with

$$
x_{i, 1}=x_{i} \quad \text { for all } i=1, \ldots, n,
$$

and

$$
a_{i j, 1}=a_{i j} \quad \text { for all } i \neq j, \quad \text { and } \quad a_{i i, 1}=2 x_{i}^{2} \quad \text { for all } i \text {. }
$$


So we have $x_{j, 1} x_{i, 1}+x_{i, 1} x_{j, 1}=a_{i j, 1}$ for all $i, j$. Let

$$
x_{1,2}:=x_{1,1} \quad \text { and } \quad x_{i, 2}:=x_{i, 1}-\frac{1}{2} a_{1 i, 1} x_{1,1}^{-2} x_{1,1} \quad \text { for all } i \geq 2 .
$$

Lemma 3.1. Retain the above notation.

(1) $x_{i, 2} x_{1,2}+x_{1,2} x_{i, 2}=0$ for all $i \geq 2$.

(2) $x_{i, 2}^{2}=x_{i, 1}^{2}-\frac{1}{4} a_{1 i, 1}^{2} x_{1,1}^{-2}$ for all $i \geq 2$.

(3) $x_{i, 2} x_{j, 2}+x_{j, 2} x_{i, 2}=a_{i j, 1}-\frac{1}{2} a_{1 i, 1} a_{1 j, 1} x_{1,1}^{-2}$ for all $2 \leq i<j \leq n$.

(4) Let $M_{2}$ be the matrix $\left(x_{i, 2} x_{j, 2}+x_{j, 2} x_{i, 2}\right)_{1 \leq i, j \leq n}$. Then $\operatorname{det} M_{2}=\operatorname{det} M_{1}$.

(5) Let

$$
C_{1}=\left\{x_{1,1}^{2 i}\right\}_{i \geq 1} .
$$

Then the localization $V_{n}(\mathcal{A})\left[C_{1}^{-1}\right]$ is free over $Z\left[C_{1}^{-1}\right]$ with basis $\left\{x_{1,2}^{d_{1}} \cdots x_{n, 2}^{d_{n}} \mid\right.$ $\left.d_{s}=0,1\right\}$.

Proof. (1)-(3) These follow by direct computation.

(4) Let $N$ be the matrix

$$
\left(\begin{array}{ccccc}
1 & 0 & 0 & \ldots & 0 \\
-\frac{1}{2} a_{12,1} x_{1,1}^{-2} & 1 & 0 & \ldots & 0 \\
-\frac{1}{2} a_{13,1} x_{1,1}^{-2} & 0 & 1 & \ldots & 0 \\
\vdots & \vdots & \vdots & \ddots & \vdots \\
-\frac{1}{2} a_{1 n, 1} x_{1,1}^{-2} & 0 & 0 & \ldots & 1
\end{array}\right) .
$$

By linear algebra and part (3), one can check that $N M_{1} N^{T}=M_{2}$. Since $\operatorname{det} N=1$, we have $\operatorname{det} M_{2}=\operatorname{det} M_{1}$.

(5) First of all, $V_{n}(\mathcal{A})$ is free over $Z$ with basis $\left\{x_{1,1}^{d_{1}} \cdots x_{n, 1}^{d_{n}} \mid d_{s}=0,1\right\}$. In the localization $V_{n}(\mathcal{A})\left[C_{1}^{-1}\right]$, this basis can be transformed to a basis $\left\{x_{1,2}^{d_{1}} \cdots x_{n, 2}^{d_{n}} \mid\right.$ $\left.d_{s}=0,1\right\}$ by using (E3.0.2).

After we have $x_{i, 2}$, define $a_{i j, 2}$ to be $x_{i, 2} x_{j, 2}+x_{j, 2} x_{i, 2}$ for all $i, j$. Now we define $x_{i, s}$ and $a_{i j, s}$ inductively.

Definition 3.2. Let $s \geq 3$ and suppose that $x_{i, s-1}$ and $a_{i j, s-1}$ are defined inductively. Define

$$
\begin{array}{ll}
x_{i, s}:=x_{i, s-1} & \text { for all } i<s, \\
x_{i, s}:=x_{i, s-1}-\frac{1}{2} a_{s-1 i, s-1} x_{s-1, s-1}^{-1} & \text { for all } i \geq s .
\end{array}
$$

Define $a_{i j, s}:=x_{i, s} x_{j, s}+x_{j, s} x_{i, s}$ for all $i, j$. 
Similar to Lemma 3.1, we have the following lemma. Its proof is also similar to the proof of Lemma 3.1, so it is omitted.

Lemma 3.3. Retain the above notation. Let $2 \leq s \leq n$.

(1) $x_{i, s} x_{j, s}+x_{j, s} x_{i, s}=0$ for all $i<j$ and $i<s$.

(2) $x_{i, s}=x_{i, s-1}$ if $i<s$ and $x_{i, s}^{2}=x_{i, s-1}^{2}-\frac{1}{4} a_{s-1 i, s-1}^{2} x_{s-1, s-1}^{-2}$ for all $i \geq s$.

(3) $x_{i, s} x_{j, s}+x_{j, s} x_{i, s}=a_{i j, s-1}-\frac{1}{2} a_{s-1 i, s-1} a_{s-1 j, s-1} x_{s-1, s-1}^{-2}$ for all $s \leq i<j \leq n$.

(4) Let $M_{s}$ be the matrix $\left(x_{i, s} x_{j, s}+x_{j, s} x_{i, s}\right)_{1 \leq i, j \leq n}$. Then $\operatorname{det} M_{s}=\operatorname{det} M_{1}$.

(5) Let $C_{s-1}$ be the Ore set

$$
\left\{x_{1,1}^{2 i_{1}} x_{2,2}^{2 i_{1}} \cdots x_{s-1, s-1}^{2 i_{s-1}}\right\}_{i_{1}, \ldots, i_{s-1} \geq 1} .
$$

Then the localization $V_{n}(\mathcal{A})\left[C_{s-1}^{-1}\right]$ is free over $Z\left[C_{s-1}^{-1}\right]$ with basis $\left\{x_{1, s}^{d_{1}} \cdots x_{n, s}^{d_{n}} \mid\right.$ $\left.d_{s}=0,1\right\}$.

We need two more lemmas before we prove the main result.

Lemma 3.4. Let $T$ be a commutative domain. Let A be a T-algebra containing $T$ as a subalgebra, generated by $x_{1}, \ldots, x_{n}$ and satisfying the relations $x_{j} x_{i}+x_{i} x_{j}=0$ for all $i<j$ and $x_{i}^{2}=a_{i} \in T$. Suppose that $A$ is a free module over $T$ with basis $\left\{x_{1}^{d_{1}} \cdots x_{n}^{d_{n}} \mid d_{s}=0,1\right\}$. Then

$$
d(A / T)=_{T^{\times}}\left(\prod_{i=1}^{n} 2 x_{i}^{2}\right)^{2^{n-1}}={ }_{T^{\times}}\left(\prod_{i=1}^{n} x_{i}^{2}\right)^{2^{n-1}} .
$$

Proof. Let $B=T_{-1}\left[x_{1}, \ldots, x_{n}\right]$ and $Z=T\left[x_{1}^{2}, \ldots, x_{n}^{2}\right]$. Then $B$ is a free module over $Z$ with basis $\left\{x_{1}^{d_{1}} \cdots x_{n}^{d_{n}} \mid d_{s}=0,1\right\}$. Let $g$ be the algebra map from $B$ to $A$ sending $T$ to $T, x_{i}$ to $x_{i}$. Then the hypotheses in Lemma 1.2 holds. By Lemma 1.2, $g(d(B / Z))={ }_{T^{\times}} d(A / T)$. Note that $d(B / Z)$ was computed in Proposition 1.4(3) to be $\left(\prod_{i=1}^{n} 2 x_{i}^{2}\right)^{2^{n-1}}$, as we assume that 2 is invertible. Now the assertion follows.

Let $A$ be an Ore domain and let $Q(A)$ denote the skew field of fractions of $A$. Let $Z$ be the commutative subalgebra $T\left[x_{1}^{2}, \ldots, x_{n}^{2}\right] \subset V_{n}(\mathcal{A})$. For each $1 \leq 1 \leq n$, let $Z_{i}$ be the subring of $Q(Z)$ of the form

$$
Q\left(T\left[x_{1}^{2}, \ldots, \widehat{x_{i}^{2}}, \ldots, x_{n}^{2}\right]\right)\left[x_{i}^{2}\right] .
$$

Lemma 3.5. Retain the above notation.

(1) $\bigcap_{i=1}^{n} Z_{i}=Q(T)\left[x_{1}^{2}, \ldots, x_{n}^{2}\right]$.

(2) $Z\left[C_{n-1}^{-1}\right] \subseteq Z_{n}$, where $Z\left[C_{n-1}^{-1}\right]$ is defined in Lemma 3.3(5).

Proof. (1) This is an easy commutative algebra fact.

(2) By Lemma 3.3(2) and induction, each $x_{i, s}^{2}$, for all $1 \leq i<n$ and all $1 \leq s \leq n$, is in $Q\left(T\left[x_{1}^{2}, \ldots, x_{n-1}^{2}\right]\right)$. So $Z\left[C_{n-1}^{-1}\right] \subseteq Z_{n}$. 
Theorem 3.6. Suppose 2 is invertible. Let $Z=T\left[x_{1}^{2}, \ldots, x_{n}^{2}\right]$. Then

$$
d\left(V_{n}(\mathcal{A}) / Z\right)=_{T^{\times}}\left(\operatorname{det} M_{1}\right)^{2^{n-1}},
$$

where $M_{1}$ is given in (E3.0.1).

Proof. Consider the variables $\left\{x_{i, n}\right\}_{i=1}^{n}$ defined in Lemma 3.3. By Lemma 3.3(5), $V_{n}(\mathcal{A})\left[C_{n-1}^{-1}\right]$ is free over $Z\left[C_{n-1}^{-1}\right]$ with basis $\left\{x_{1, s}^{d_{1}} \cdots x_{n, s}^{d_{n}} \mid d_{s}=0,1\right\}$. By Lemma 3.4 , the discriminant

$$
d\left(V_{n}(\mathcal{A})\left[C_{n-1}^{-1}\right] / Z\left[C_{n-1}^{-1}\right]\right)
$$

is of the form $\left(\prod_{i=1}^{n} x_{i}^{2}\right)^{2^{n-1}}$ up to a unit in $Z\left[C_{n-1}^{-1}\right]$. By Lemma 3.3(4), we have

$$
d\left(V_{n}(\mathcal{A})\left[C_{n-1}^{-1}\right] / Z\left[C_{n-1}^{-1}\right]\right)=\left(\prod_{i=1}^{n} x_{i}^{2}\right)^{2^{n-1}}=\left(\operatorname{det} M_{n}\right)^{2^{n-1}}=\left(\operatorname{det} M_{1}\right)^{2^{n-1}}
$$

By Lemma 1.3,

$$
d\left(V_{n}(\mathcal{A}) / Z\right)=_{\left(Z\left[C_{n-1}^{-1}\right]\right)} \times d\left(V_{n}(\mathcal{A})\left[C_{n-1}^{-1}\right] / Z\left[C_{n-1}^{-1}\right]\right)=_{\left(Z\left[C_{n-1}^{-1}\right]\right)} \times\left(\operatorname{det} M_{1}\right)^{2^{n-1}} .
$$

Let $\Phi$ be the element $d\left(V_{n}(\mathcal{A}) / Z\right)^{-1}\left(\operatorname{det} M_{1}\right)^{2^{n-1}}$. Then $\Phi \in\left(Z\left[C_{n-1}^{-1}\right]\right)^{\times}$. This means that both $\Phi$ and $\Phi^{-1}$ are in $Z\left[C_{n-1}^{-1}\right] \subseteq Z_{n}$. By symmetry, $\Phi$ is $Z_{i}$ for all $i$. Thus $\Phi$ is in $\bigcap_{i=1}^{n} Z_{i}=Q(T)\left[x_{1}^{2}, \ldots, x_{n}^{2}\right]$. Similarly, $\Phi^{-1}$ is in $Q(T)\left[x_{1}^{2}, \ldots, x_{n}^{2}\right]$. Therefore, $\Phi, \Phi^{-1} \in Q(T)$.

Write $d\left(V_{n}(\mathcal{A}) / Z\right)=c\left(\operatorname{det} M_{1}\right)^{2^{n-1}}$, where $c=\Phi^{-1} \in Q(T)$. It remains to show $c \in Z^{\times}$. Note that $V_{n}(\mathcal{A})$ is a filtered algebra such that $\operatorname{gr} V_{n}(\mathcal{A}) \cong T_{-1}\left[x_{1}, \ldots, x_{n}\right]$. By Lemma 1.5,

$$
\operatorname{gr} d\left(V_{n}(\mathcal{A}) / Z\right)={ }_{Z^{\times}} d\left(\operatorname{gr} V_{n}(\mathcal{A}) / \operatorname{gr} Z\right) .
$$

The left-hand side of the above is $c\left(\prod_{i=1} x_{i}^{2}\right)^{2^{n-1}}$ and the right-hand side of the above is $\left(\prod_{i=1} x_{i}^{2}\right)^{2^{n-1}}$ by Proposition 1.4(3) (assuming 2 is invertible). Thus $c \in Z^{\times}$, as required.

Theorem 0.2 is a special case of Theorem 3.6 by taking $a_{i j}=1$ for all $i<j$.

The algebras $V_{n}(\mathcal{A})$ and $W_{n}$ are special Clifford algebras. Now we consider a Clifford algebra in a more general setting. Let $T$ be a commutative domain and let $V$ be a free $T$-module of rank $n$. Given a quadratic form $q: V \rightarrow T$, we can associate to this data the Clifford algebra

$$
C(V, q)=\frac{T\langle V\rangle}{\left(x^{2}-q(x) \mid x \in V\right)} .
$$


Note that this $q$ is different from the parameter $q$ in the definition of the $q$-quantum Weyl algebra $A_{q}$ and the parameter set $\boldsymbol{q}$ in the $V_{n}(\boldsymbol{q}, \mathcal{A})$ and $T_{\boldsymbol{q}}\left[x_{1}, \ldots, x_{n}\right]$. Consider the bilinear form associated to $q$,

$$
b(x, y)=\frac{1}{2}(q(x+y)-q(x)-q(y))
$$

for all $x, y \in V$. If we choose a $T$-basis $x_{1}, \ldots, x_{n}$ for $V$ and let

$$
\mathfrak{B}:=\left(b_{i j}\right)=\left(b\left(x_{i}, x_{j}\right)\right)_{n \times n} \in T^{n \times n}
$$

be the symmetric matrix which represents $b$ with respect to this basis, then the relations of $C(V, q)$ are

$$
x_{i} x_{j}+x_{j} x_{i}=2 b_{i j} \quad \text { for all } i, j .
$$

Define $\operatorname{det}(q)$ to be $\operatorname{det}(\mathfrak{B})$.

The following main result is a consequence of Theorem 3.6 and Lemma 1.2.

Theorem 3.7. Let $A:=C(V, q)$ be a Clifford algebra over a commutative domain $T$ defined by a quadratic form $q: V \rightarrow T$. Pick a T-basis of $V$, say $\left\{x_{i}\right\}_{i=1}^{n}$. Then

$$
d(A / T)=_{T^{\times}}\left(\operatorname{det}\left(x_{i} x_{j}+x_{j} x_{i}\right)_{n \times n}\right)^{2^{n-1}}=_{T^{\times}} \operatorname{det}(q)^{2^{n-1}} .
$$

Proof. Let $b: V^{\otimes 2} \rightarrow T$ be the symmetric bilinear form associated to the quadratic form $q$. Let $a_{i j}=2 b\left(x_{i}, x_{j}\right)$ for all $i<j$ and $\mathcal{A}=\left\{a_{i j}\right\}_{1 \leq i<j \leq n}$. Then there is a canonical algebra surjection $\pi: V_{n}(\mathcal{A}) \rightarrow C(V, q)$ sending $x_{i} \rightarrow x_{i}$ for all $i=1, \ldots, n$ and $t \rightarrow t$ for all $t \in T$, and the kernel of $\pi$ is the ideal generated by $\left\{x_{i}^{2}-b_{i i}\right\}_{i=1}^{n}$. Clearly, $\pi\left(T\left[x_{1}^{2}, \ldots, x_{n}^{2}\right]\right)=T$ and the matrix $\left(x_{i} x_{j}+x_{j} x_{i}\right)_{n \times n}$ equals $M_{1}$. It is easy to check that $\left\{x_{1}^{d_{1}} \cdots x_{n}^{d_{n}} \mid d_{i}=0,1\right\}$ is a basis of $V_{n}(\mathcal{A})$ over $T\left[x_{1}^{2}, \ldots, x_{n}^{2}\right]$ and a basis of $C(V, q)$ over $T$. The first equation of (E3.7.1) follows from Theorem 3.6 and Lemma 1.2 and the second equation follows from the fact that $2 \mathfrak{B}=\left(x_{i} x_{j}+x_{j} x_{i}\right)_{n \times n}$ and 2 is invertible.

In the rest of this section we briefly discuss "generic Clifford algebras", which will appear again in Section 8. (This generic Clifford algebra should be called a "universal Clifford algebra", but the term "universal Clifford algebra" has already been used).

Fix an integer $n$. Let $I$ be the set $\{(i, j) \mid 1 \leq i \leq j \leq n\}$ that can be thought of as the quotient set $\{(i, j) \mid 1 \leq i, j \leq n\} /((i, j) \sim(j, i))$. Let $w$ denote the integer $\frac{1}{2} n(n+1)$. There is a bijection between $I$ and the set of the first $w$ integers $\{1,2, \ldots, w\}$. Let $T_{g}$ be the commutative domain $k\left[t_{(i, j)} \mid(i, j) \in I\right]$, which is isomorphic to $k\left[t_{1}, \ldots, t_{w}\right]$. Define a $T_{g}$-algebra $A_{g}$ generated by $x_{1}, \ldots, x_{n}$ and subject to the relations

$$
x_{i} x_{j}+x_{j} x_{i}=2 t_{(i, j)} \quad \text { for all } 1 \leq i \leq j \leq n .
$$


Let $V_{g}=\bigoplus_{i=1}^{n} T_{g} x_{i}$. Define a bilinear form $b_{g}: V_{g} \otimes V_{g} \rightarrow T_{g}$ by $b_{g}\left(x_{i}, x_{j}\right)=t_{(i, j)}$ and the associated quadratic form by $q_{g}(x)=b_{g}(x, x)$ for all $x \in V_{g}$. The "generic Clifford algebra" $A_{g}$ is defined to be the Clifford algebra associated to $\left(V_{g}, q_{g}\right)$. For any Clifford algebra $C(V, q)$ over a commutative ring $T$, by comparing (E3.6.3) with (E3.7.2), one sees that there is an algebra map $A_{g} \rightarrow C(V, q)$ sending $x_{i} \rightarrow x_{i}$ and $t_{(i, j)} \rightarrow b_{i j}$. Define $\operatorname{deg} x_{i}=1$ for all $i$ and $\operatorname{deg} t_{(i, j)}=2$ for all $(i, j) \in I$. Then $A_{g}$ is a connected graded algebra over $k$.

We also define some factor algebras of $A_{g}$. Let $J$ be a subset of $\{(i, j) \mid$ $1 \leq i<j \leq n\}$ and let $w_{J}$ denote the integer $w-|J|$. Let $T_{g, J}$ be the commutative polynomial ring $k\left[t_{i, j} \mid(i, j) \in I \backslash J\right]$, which is isomorphic to $k\left[t_{1}, \ldots, t_{w_{J}}\right]$. Define a $T_{g, J}$-algebra $A_{g, J}$ generated by $x_{1}, \ldots, x_{n}$ and subject to the relations

$$
x_{i} x_{j}+x_{j} x_{i}= \begin{cases}2 t_{(i, j)}, & (i, j) \in I \backslash J, \\ 0, & (i, j) \in J .\end{cases}
$$

Let $V_{g, J}=\bigoplus_{i=1}^{n} T_{g, J} x_{i}$. Define a bilinear form $b_{g, J}: V_{g, J} \otimes V_{g, J} \rightarrow T_{g, J}$ by

$$
b_{g, J}\left(x_{i}, x_{j}\right)= \begin{cases}t_{(i, j)}, & (i, j) \in I \backslash J, \\ 0, & (i, j) \in J\end{cases}
$$

and the associated quadratic form by $q_{g, J}(x)=b_{g}(x, x)$ for all $x \in V_{g, J}$. Then $A_{g, J}$ is the Clifford algebra associated to $\left(V_{g, J}, q_{g, J}\right)$. If $J \subseteq J^{\prime} \subseteq\{(i, j) \mid 1 \leq i<j \leq n\}$, there is an algebra map $A_{g, J} \rightarrow A_{g, J^{\prime}}$ sending $x_{i} \rightarrow x_{i}$ and

$$
t_{(i, j)} \rightarrow \begin{cases}t_{(i, j)}, & (i, j) \notin J^{\prime} \\ 0, & (i, j) \in J^{\prime} \backslash J .\end{cases}
$$

In particular, $A_{g, J}$ is a connected graded factor ring of $A_{g}$.

In part (4) of the next lemma, we will use a few undefined concepts that are related to the homological properties of an algebra. We refer to [Levasseur 1992; Lu et al. 2007; Rogalski and Zhang 2012] for definitions.

Lemma 3.8. Retain the above notation. Assume that $k$ is a field of characteristic not 2. Let $J^{\prime}$ be subset of $\{(i, j) \mid 1 \leq i<j \leq n\}$ and let $J=J^{\prime} \backslash\left\{\left(i_{0}, j_{0}\right)\right\}$ for some $\left(i_{0}, j_{0}\right) \in J^{\prime}$.

(1) The Hilbert series of $A_{g}$ is

$$
H_{A_{g}}(t)=\frac{(1+t)^{n}}{\left(1-t^{2}\right)^{w}}, \quad \text { where } w=\frac{1}{2} n(n+1) .
$$

(2) The Hilbert series of $A_{g, J}$ is

$$
H_{A_{g, J}}(t)=\frac{(1+t)^{n}}{\left(1-t^{2}\right)^{w_{J}}}, \quad \text { where } w_{J}=w-|J| .
$$

(3) $t_{\left(i_{0}, j_{0}\right)}$ is a central regular element in $A_{g, J^{\prime}}$, and $A_{g, J}=A_{g, J^{\prime}} /\left(t_{\left(i_{0}, j_{0}\right)}\right)$. 
(4) $A_{g}$ and $A_{g, J}$ are connected graded Artin-Schelter regular, Auslander regular, Cohen-Macaulay noetherian domains.

Proof. (1) Note that $A_{g}$ is a free module over $T_{g}$ with basis $\left\{x_{1}^{d_{1}} \cdots x_{n}^{d_{n}} \mid d_{s}=0,1\right\}$. Recall that $\operatorname{deg} x_{i}=1$ and $\operatorname{deg} t_{(i, j)}=2$. We have

$$
H_{A_{g}}(t)=(1+t)^{n} H_{T_{g}}(t)=\frac{(1+t)^{n}}{\left(1-t^{2}\right)^{w}} .
$$

(2) The proof is similar. Use the fact that $H_{T_{g, J}}(t)=1 /\left(1-t^{2}\right)^{w_{J}}$.

(3) It is clear that $t_{\left(i_{0}, j_{0}\right)}$ is central in $A_{g, J^{\prime}}$ and that $A_{g, J}=A_{g, J^{\prime}} /\left(t_{\left(i_{0}, j_{0}\right)}\right)$. So the ideal $\left(t_{\left(i_{0}, j_{0}\right)}\right)$ is the left ideal $t_{\left(i_{0}, j_{0}\right)} A_{g, J^{\prime}}$ and the right ideal $A_{g, J^{\prime}} t_{\left(i_{0}, j_{0}\right)}$. By parts (1) and (2), the Hilbert series of $\left(t_{\left(i_{0}, j_{0}\right)}\right)$ is $t^{2} H_{A_{g, J^{\prime}}}(t)$. So $t_{\left(i_{0}, j_{0}\right)}$ is regular.

(4) We only provide a proof for $A_{g}$. The proof for $A_{g, J}$ is similar.

From part (3), $J_{M}:=\left\{t_{(i, j)} \mid 1 \leq i<j \leq n\right\}$ is a sequence of regular central elements in $A_{g}$ of positive degree. It is easy to see that $A_{g, J_{M}}\left(=A_{g} /\left(J_{M}\right)\right)$ is isomorphic to the skew polynomial ring $k_{-1}\left[x_{1}, \ldots, x_{n}\right]$, which is an Artin-Schelter regular, Auslander regular, Cohen-Macaulay noetherian domain. Applying [Lu et al. 2007, Lemma 7.6] repeatedly, $A_{g}$ has finite global dimension. Applying [Levasseur 1992, Proposition 3.5, Theorem 5.10] repeatedly, $A_{g}$ is a noetherian Auslander Gorenstein and Cohen-Macaulay domain. By [Levasseur 1992, Theorem 6.3], $A_{g}$ is ArtinSchelter Gorenstein. Since $A_{g}$ has finite global dimension, it is Auslander regular and Artin-Schelter regular.

Remark 3.9. Retain the above notation. (1) Some homological properties of the algebra $A_{g}$ are given in Lemma 3.8. It would be interesting to work out combinatorial and geometric invariants (and properties) of $A_{g}$. For example, what are the pointmodule and line-module schemes of $A_{g}$ ? Definitions of these schemes can be found in [Vancliff and Van Rompay 2000; Vancliff et al. 1998].

(2) Another way of presenting $A_{g}$ is the following. Let $S$ be a $k$-vector space of dimension $n$. Define $A_{g}$ to be $k\langle S\rangle /\left(\left[x^{2}, y\right]=0 \mid\right.$ for all $\left.x, y, \in S\right)$. By using this new expression, one can easily see that the group of graded algebra automorphisms of $A_{g}$, denoted by $\operatorname{Aut}_{\mathrm{gr}}\left(A_{g}\right)$, is isomorphic to $\mathrm{GL}_{n}(k)$.

(3) Suppose $n \geq 2$. The full automorphism group $\operatorname{Aut}\left(A_{g}\right)$ has not been determined. It is known that $\operatorname{Aut}\left(A_{g}\right)$ is not affine. For example, if $f(t)$ is a polynomial in $t$, then

$$
x_{i} \rightarrow \begin{cases}x_{i}, & i>1 \\ x_{1}+f\left(\left[x_{1}, x_{2}\right]^{2}\right) x_{2}, & i=1\end{cases}
$$

extends to an algebra automorphism of $A_{g}$.

(4) It seems interesting to study the "cubic algebra" $k\langle S\rangle /\left(\left[x^{3}, y\right]=0 \mid\right.$ for all $\left.x, y \in S\right)$ and higher-degree analogues. 
(5) The quotient division ring of $A_{g}$, denoted by $D_{g}$, is called the "generic Clifford division algebra of rank $n$ ". It would be interesting to study algebraic properties or invariants of $D_{g}$.

\section{Center of skew polynomial rings}

To use the discriminant most effectively, one needs to first understand the center of an algebra. In this section we give a criterion for when $T_{q}\left[x_{1}, \ldots, x_{n}\right]$ is free over its center and when the center of $T_{q}\left[x_{1}, \ldots, x_{n}\right]$ is a polynomial ring.

Recall that $T$ is a commutative domain and $\boldsymbol{q}:=\left\{q_{i j} \in T^{\times} \mid 1 \leq i<j \leq n\right\}$ is a set of invertible scalars. Let $P:=T_{q}\left[x_{1}, \ldots, x_{n}\right]$ be the skew polynomial ring over $T$ and subject to the relations (E0.2.1). We assume that $d_{i j}:=o\left(q_{i j}\right)<\infty$ and write

$$
q_{i j}=\exp \left(2 \pi \sqrt{-1} k_{i j} / d_{i j}\right),
$$

where $\left|k_{i j}\right|<d_{i j}$ and $\left(k_{i j}, d_{i j}\right)=1$. Note that, by our convention, $q_{i j}=q_{j i}^{-1}$ for all $i, j$. Hence, we choose $k_{i j}=-k_{j i}$ and $d_{i j}=d_{j i}$. We also adopt the convention that if $q_{i j}=1$ then $k_{i j}=0$ and $d_{i j}=1$. In particular, $k_{i i}=0$ and $d_{i i}=1$. We can extend $P$ to $P\left[x_{1}^{-1}, \ldots, x_{n}^{-1}\right]$, with an inverse for each $x_{i}$, with the expected relations

$$
x_{i} x_{i}^{-1}=x_{i}^{-1} x_{i}=1, \quad x_{j} x_{i}^{-1}=q_{i j}^{-1} x_{i}^{-1} x_{j}, \quad \text { and } \quad x_{j}^{-1} x_{i}^{-1}=q_{i j} x_{i}^{-1} x_{j}^{-1} .
$$

We need to do some analysis to understand the center of $P$. Let $\eta_{i}$ denote conjugation by $x_{i}$, sending $f \mapsto x_{i}^{-1} f x_{i}$, and let $\xi=x_{1}^{s_{1}} \cdots x_{n}^{s_{n}}$. Then

$$
\eta_{i}(\xi)=\exp \left(2 \pi \sqrt{-1} e_{i}^{T} Y s\right) \xi
$$

where $Y \in \mathfrak{s o}_{n}(\mathbb{Q})$ has $(i, j)$-th entry $k_{i j} / d_{i j}, s$ is the column vector whose $i$-th entry is $s_{i}$ appearing in the powers of $\xi$, and $\boldsymbol{e}_{i}$ is the $i$-th standard basis vector in $\mathbb{Q}^{n}$.

Lemma 4.1. Retain the above notation. Then $\xi$ is in the center $Z(P)$ of $P$ if and only if $Y s \in \mathbb{Z}^{n}$.

Proof. Since $P$ is generated by $\left\{x_{i}\right\}$, we have $\xi \in Z(P)$ if and only if $\eta_{i}(\xi)=\xi$ for all $i$, if and only if $\exp \left(2 \pi \sqrt{-1} \boldsymbol{e}_{i}^{T} Y \boldsymbol{s}\right)=1$, if and only if $\boldsymbol{e}_{i}^{T} Y \boldsymbol{s} \in \mathbb{Z}$ for all $i$, and finally, if and only if $Y s \in \mathbb{Z}^{n}$.

By choosing the standard basis for $\mathbb{Q}^{n}$, we can consider $Y$ as a linear transformation $\mathbb{Q}^{n} \rightarrow \mathbb{Q}^{n}$ by sending $\boldsymbol{s} \mapsto Y \boldsymbol{s}$. Here we view $\mathbb{Q}^{n}$ as column vectors and $Y$ as a left multiplication. We can restrict this map to $\mathbb{Z}^{n} \subset \mathbb{Q}^{n}$ (embedded via the standard basis) and compose with the quotient $\mathbb{Q}^{n} \rightarrow \mathbb{Q}^{n} / \mathbb{Z}^{n}$ to obtain a $\mathbb{Z}$-module homomorphism $Y^{\prime}: \mathbb{Z}^{n} \rightarrow \mathbb{Q}^{n} / \mathbb{Z}^{n}$.

Lemma 4.2. Retain the above notation. Then $\xi \in Z(P)$ if and only if $s \in \operatorname{ker}\left(Y^{\prime}\right)$.

Proof. By Lemma 4.1, $\xi \in Z(P)$ if and only if $Y s \in \mathbb{Z}^{n}$, which is equivalent to $Y^{\prime}(s)=0$ by the definition of $Y^{\prime}$. 
Let $D$ be the matrix $\left(d_{i j}\right)_{n \times n}$ and let $L_{i}$ be the lcm of the entries in the $i$-th row of $D$, namely, $L_{i}=\operatorname{lcm}\left\{d_{i j} \mid j=1, \ldots, n\right\}$. Since $D$ is a symmetric matrix, $L_{i}$ is also the $\mathrm{lcm}$ of the entries in $i$-th column. Observe that $Z(P)$ contains the central subring $P^{\prime}:=k\left[x_{1}^{L_{1}}, \ldots, x_{n}^{L_{n}}\right]$. In other words, $\operatorname{ker}\left(Y^{\prime}\right)$ contains the $\mathbb{Z}$-lattice $\Lambda$ spanned by $L_{i} \boldsymbol{e}_{i}$ for $i=1, \ldots, n$. Therefore, $Y^{\prime}$ factors through

$$
\mathbb{Z}^{n} \rightarrow M:=\mathbb{Z}^{n} / \Lambda=\bigoplus_{i=1}^{n} \mathbb{Z} / L_{i} \mathbb{Z} .
$$

For each $s \in \mathbb{Z}^{n}$, the $i$-th entry of $Y^{\prime}(s)$ is $\sum_{j} k_{i j} s_{j} / d_{i j} \in \mathbb{Q} / \mathbb{Z}$, which is $L_{i}$-torsion, or equivalently, in $L_{i}^{-1} \mathbb{Z} / \mathbb{Z}$. Therefore, $Y^{\prime}$ induces a map

$$
M \rightarrow M^{\prime}:=\bigoplus_{i=1}^{n} L_{i}^{-1} \mathbb{Z} / \mathbb{Z} .
$$

Since $M^{\prime}$ is naturally isomorphic to $M$, we can define an endomorphism

$$
\bar{Y}: M \rightarrow M
$$

by setting

$$
\bar{Y} \boldsymbol{s}=\left(\sum_{j=1}^{n} L_{i}\left(k_{i j} s_{j} / d_{i j}\right)\right)_{i=1}^{n} .
$$

In particular, $\bar{Y} \boldsymbol{e}_{j}=\sum_{i=1}^{n}\left(k_{i j} L_{i} / d_{i j}\right) \boldsymbol{e}_{i}$. Sometimes we think of $\bar{Y}$ as a matrix:

$$
\bar{Y}=\left(k_{i j} L_{i} / d_{i j}\right)_{n \times n}=\operatorname{diag}\left(L_{1}, \ldots, L_{n}\right) Y .
$$

The following lemma is a reinterpretation of [CPWZ 2016, Lemma 2.3].

Lemma 4.3. Retain the above notation. The following are equivalent.

(1) The center $Z(P)$ of $P$ is a polynomial ring.

(2) $Z(P)=P^{\prime}$.

(3) $\operatorname{ker}(\bar{Y})=0$.

(4) $\bar{Y}$ is an isomorphism.

Proof. (1) $\Leftrightarrow(2)$ : One implication is clear. For the other implication, we assume that the center $Z(P)$ is a polynomial ring. By [CPWZ 2016, Lemma 2.3], $Z(P)$ is of the form $T\left[x_{1}^{a_{i}}, \ldots, x_{n}^{a_{i}}\right]$. It is easy to check that $L_{i} \mid a_{i}$ for all $i$. Since $Z(P) \supseteq P^{\prime}$, $a_{i}=L_{i}$ for all $i$. The assertion follows.

(3) $\Rightarrow(2)$ : Let $\xi:=x_{1}^{s_{1}} \cdots x_{n}^{s_{n}} \in Z(P)$ and $s=\left(s_{i}\right)_{i=1}^{n}$. By Lemma 4.2, $s \in \operatorname{ker}\left(Y^{\prime}\right)$. Since $\bar{Y}$ is induced by $Y^{\prime}$, we have $\bar{Y}(s)=0$. By part (3), $s=0$ in $M=\mathbb{Z}^{n} / \Lambda$. So $s \in \Lambda$, which is equivalent to $\xi \in P^{\prime}$. Therefore, $Z(P)=P^{\prime}$, as desired.

(2) $\Rightarrow(3)$ : Let $\xi:=x_{1}^{s_{1}} \cdots x_{n}^{s_{n}} \in P$, where $s:=\left(s_{i}\right)_{i=1}^{n} \in \operatorname{ker}(\bar{Y})$, viewed as a vector in $M$. By the definition of $M$, we might assume that each $s_{i}$ is nonnegative and less 
than $L_{i}$. Since $\bar{Y}$ is induced by $Y^{\prime}$, we have $s \in \operatorname{ker}\left(Y^{\prime}\right)$. By Lemma 4.2, $\xi \in Z(P)$. By part (2) and our choice of $0 \leq s_{i}<L_{i}$, we have $\xi=1$ or $s=0$, as desired.

(3) $\Leftrightarrow(4)$ : This is clear since $M$ is finite.

The advantage of working with $\bar{Y}$ is that $\operatorname{ker}(\bar{Y})=0$ is equivalent to $\bar{Y}$ being an isomorphism. Next we need to understand when $\bar{Y}$ is an isomorphism. For the rest of this section we use $\otimes$ for $\otimes_{\mathbb{Z}}$ and $\mathbb{F}_{p}$ for $\mathbb{Z} / p \mathbb{Z}$.

Lemma 4.4. The morphism $\bar{Y}$ is an isomorphism if and only if $\bar{Y} \otimes \mathbb{F}_{p}$ is an isomorphism for all primes $p$.

Proof. As a $\mathbb{Z}$-module, $M$ is finite, and it suffices to show that $\bar{Y}$ is surjective if and only if $\bar{Y} \otimes \mathbb{F}_{p}$ is surjective for each prime $p$. This is clear since $-\otimes \mathbb{F}_{p}$ is right exact, so surjectivity of a map can be checked on closed fibers.

Fix any prime $p$. Let $M_{p}=M \otimes \mathbb{F}_{p}$ and $\bar{Y}_{p}=\bar{Y} \otimes \mathbb{F}_{p}$. For any $\boldsymbol{e}_{i}$, if $L_{i} \notin p \mathbb{Z}$, then the image of $\boldsymbol{e}_{i}$ is zero in $M_{p}$. We can therefore use $\left\{\boldsymbol{e}_{i} \mid L_{i} \in p \mathbb{Z}\right\}$ as a basis of $M_{p}$. Consequently, $M_{p}$ is a vector space over $\mathbb{F}_{p}$ of dimension at most $n$, and we can write $\bar{Y}_{p}$ as a matrix over $\mathbb{F}_{p}$. Next we will decompose the vector space $M_{p}$ and the matrix $\bar{Y}_{p}$.

For each positive integer $m$, let $M_{p, m}$ denote the subspace of $M_{p}$ generated by $\left\{\boldsymbol{e}_{i} \mid L_{i} \in p^{m} \mathbb{Z}-p^{m+1} \mathbb{Z}\right\}$. Let $\bar{Y}_{p, m}$ be the endomorphism

$$
M_{p, m} \longrightarrow M_{p} \stackrel{\bar{Y}_{p}}{\longrightarrow} M_{p} \longrightarrow M_{p, m},
$$

where the first map is the inclusion and the last map is the natural projection using the given basis $\left\{\boldsymbol{e}_{i} \mid L_{i} \in p \mathbb{Z}\right\}$. Then $\bar{Y}_{p, m}$ can be expressed as the submatrix of $\bar{Y}$ taken from the rows and columns with indices $i$ such that $\boldsymbol{e}_{i} \in M_{p, m}$. For all but finitely many values of $m$, we have $M_{p, m}=0$, and in this case, $\bar{Y}_{p, m}$ is a $0 \times 0$ matrix. We adopt the convention that the determinant of a $0 \times 0$ matrix is 1 . In general, $\operatorname{det}\left(\bar{Y}_{p, m}\right)$ is in $\mathbb{F}_{p}$.

Lemma 4.5. The following are equivalent.

(1) The map $\bar{Y}_{p}$ is an isomorphism.

(2) For all positive integers $m, \bar{Y}_{p, m}$ is an isomorphism.

(3) $\operatorname{det}\left(\bar{Y}_{p, m}\right) \neq 0$ for all positive integers $m$.

Proof. It is clear that (2) and (3) are equivalent, so we need only show that (1) and (2) are equivalent.

Let $m>0$, and let $i, j$ be such that $L_{i} \in p^{m} \mathbb{Z}-p^{m+1} \mathbb{Z}$ and $L_{j} \notin p^{m} \mathbb{Z}$. Since $L_{j}=\operatorname{lcm}\left\{d_{k j} \mid k=1, \ldots, n\right\}$, we have $d_{i j} \notin p^{m} \mathbb{Z}$ and $k_{i j} L_{i} / d_{i j} \in p \mathbb{Z}$. Therefore, the $\boldsymbol{e}_{i}$-component of $\bar{Y}_{p} \boldsymbol{e}_{j}$ is zero. We can extend this to show that, for any $m>m^{\prime}>0$, 
the $M_{p, m^{\prime}}$-component of $\bar{Y}_{p}\left(M_{p, m}\right)$ is zero, or equivalently,

$$
\bar{Y}_{p}\left(M_{p, m}\right) \subseteq \bigoplus_{n \geq m} M_{p, n}=: N_{m}
$$

This implies that, for any $m>0, \bar{Y}_{p}$ acts as an endomorphism on $N_{m}$. Since each $M_{p}$ is finite dimensional, $\bar{Y}_{p}$ is an isomorphism if and only if it acts as an isomorphism on each subquotient $N_{m} / N_{m+1} \cong M_{p, m}$. This action is already given by $\bar{Y}_{p, m}$, so the assertion follows.

Combining all the lemmas in this section we have:

Theorem 4.6. The center of the skew polynomial ring $T_{q}\left[x_{1}, \ldots, x_{n}\right]$ is a polynomial ring if and only if $\operatorname{det}\left(\bar{Y}_{p, m}\right) \neq 0$ for all primes $p$ and all integers $m>0$.

Theorem 4.6 is a slight generalization of Theorem 0.3(a) without the hypothesis that $q_{i j} \neq 1$ for all $i \neq j$. The definition of the matrices $\bar{Y}_{p, m}$ is not straightforward, so we give an example below. Hopefully, the example will show that this matrix is not hard to understand.

Example 4.7. We start with the following skew-symmetric matrix with entries in $\mathbb{Q}$ :

$$
Y:=\left(\begin{array}{rrrrrr}
0 & \frac{4}{27} & \frac{2}{9} & 0 & \frac{2}{3} & \frac{3}{5} \\
-\frac{4}{27} & 0 & \frac{1}{3} & \frac{7}{9} & \frac{1}{3} & \frac{1}{5} \\
-\frac{2}{9} & -\frac{1}{3} & 0 & \frac{1}{6} & \frac{1}{2} & \frac{1}{2} \\
0 & -\frac{7}{9} & -\frac{1}{6} & 0 & \frac{2}{3} & 0 \\
-\frac{2}{3} & -\frac{1}{3} & -\frac{1}{2} & -\frac{2}{3} & 0 & \frac{5}{8} \\
-\frac{3}{5} & -\frac{1}{5} & -\frac{1}{2} & 0 & -\frac{5}{8} & 0
\end{array}\right) .
$$

One can easily construct $q_{i j}$ by (E4.0.1) and the skew polynomial ring $T_{q}\left[x_{1}, \ldots, x_{6}\right]$ by (E0.2.1), but the point of this example is to work out the matrices $\bar{Y}_{p, m}$ for all primes $p$ and all $m>0$. By considering the denominators of the entries of $Y$, one sees that

$$
\left(L_{1}, L_{2}, L_{3}, L_{4}, L_{5}, L_{6}\right)=\left(3^{3} \cdot 5,3^{3} \cdot 5,2 \cdot 3^{2}, 2 \cdot 3^{2}, 2^{3} \cdot 3,2^{3} \cdot 5\right) .
$$

This implies that $\bar{Y}_{p, m}$ is a trivial matrix (or a $0 \times 0$ matrix) except for $p=2,3,5$. Next we consider

$$
\bar{Y}=\operatorname{diag}\left(L_{1}, \ldots, L_{6}\right) Y=\left(\begin{array}{cccccc}
0 & 20 & 30 & 0 & 90 & 81 \\
-20 & 0 & 45 & 105 & 45 & 27 \\
-4 & -6 & 0 & 3 & 9 & 9 \\
0 & -14 & -3 & 0 & 12 & 0 \\
-16 & -8 & -12 & -16 & 0 & 15 \\
-24 & -8 & -20 & 0 & -25 & 0
\end{array}\right) .
$$


Recall that $M_{p, m}$ has a basis $\left\{\boldsymbol{e}_{i} \mid L_{i} \in p^{m} \mathbb{Z}-p^{m+1} \mathbb{Z}\right\}$ and $\bar{Y}_{p, m}$ is the square submatrix of $\bar{Y}$ with indices $\left\{i \mid L_{i} \in p^{m} \mathbb{Z}-p^{m+1} \mathbb{Z}\right\}$ and with entries evaluated in $\mathbb{F}_{p}$.

For $p=2, \bar{Y}_{2, m}$ are the following:

- $\bar{Y}_{2,1}$ is the principle $(3,4)$-submatrix of $Y$, and is $\left(\begin{array}{ll}0 & 1 \\ 1 & 0\end{array}\right)$.

- $\bar{Y}_{2,3}$ uses indices 5, 6, and is $\left(\begin{array}{ll}0 & 1 \\ 1 & 0\end{array}\right)$.

- For all $m=2$ or $m>3, \bar{Y}_{2, m}$ is trivial.

Therefore, $\bar{Y}_{2}$ is an isomorphism by Lemma 4.5.

For $p=3, \bar{Y}_{3, m}$ are the following:

- $\bar{Y}_{3,1}$ uses only index 5 , and is the $1 \times 1$ zero matrix.

- $\bar{Y}_{3,2}$ uses indices 3,4 , and is the $2 \times 2$ zero matrix.

- $\bar{Y}_{3,3}$ uses indices 1,2 , and is $\left(\begin{array}{rr}0 & 1 \\ -1 & 0\end{array}\right)$.

- For all $m>3, \bar{Y}_{3, m}$ is trivial.

Since $\operatorname{det}\left(\bar{Y}_{3,1}\right)=\operatorname{det}\left(\bar{Y}_{3,2}\right)=0, \bar{Y}_{3}$ is not an isomorphism by Lemma 4.5. Consequently, the center of $T_{q}\left[x_{1}, \ldots, x_{6}\right]$ is not a polynomial ring by Theorem 4.6.

For $p=5, \bar{Y}_{5, m}$ are the following:

- $\bar{Y}_{5,1}$ uses indices $1,2,6$, and

$$
\bar{Y}_{5,1}=\left(\begin{array}{rrr}
0 & 0 & 1 \\
0 & 0 & 2 \\
-1 & -2 & 0
\end{array}\right) .
$$

- For all $m>1, \bar{Y}_{5, m}$ is trivial.

It is easy to check that $\operatorname{det}\left(\bar{Y}_{5,1}\right)=0$. Therefore, $\bar{Y}_{5}$ is not an isomorphism.

For $p>5, \bar{Y}_{p, m}$ is trivial for all $m>0$.

\section{Low dimensional cases}

We start with some easy consequences of Theorem 4.6 and then discuss the case when $n$ is 3 or 4 .

Corollary 5.1. Suppose there are a prime $p$ and an $m>0$ such that $M_{p, m}$ is odd dimensional. Then $\bar{Y}_{p}$ is not an isomorphism. As a consequence, the center of $T_{q}\left[x_{1}, \ldots, x_{n}\right]$ is not a polynomial ring.

Proof. If $\bar{Y}_{p, m}$ is a skew-symmetric matrix of odd size, its determinant is zero (this is true even when $p=2$ ). The rest follows from Lemma 4.5 and Theorem 4.6.

Corollary 5.2. Suppose there is a prime $p$ such that $M_{p}$ is odd dimensional. Then $\bar{Y}_{p}$ is not an isomorphism. As a consequence, the center of $T_{q}\left[x_{1}, \ldots, x_{n}\right]$ is not a polynomial ring. 
Proof. Since $M_{p}=\bigoplus_{m=1}^{\infty} M_{p, m}$, if it is odd dimensional, at least one $M_{p, m}$ must be odd dimensional. The assertion follows from Corollary 5.1.

Corollary 5.3. Suppose, for each prime $p$, that $p \mid d_{i j}$ for at most one pair $(i, j)$, $1 \leq i<j \leq n$. Then $\bar{Y}_{p}$ is an isomorphism for each $p$. As a consequence, the center of $T_{q}\left[x_{1}, \ldots, x_{n}\right]$ is a polynomial ring.

Proof. If $d_{i j} \notin p \mathbb{Z}$ for all $i, j$, then $L_{i} \notin p \mathbb{Z}$ for all $i, M_{p}=0$ and $\bar{Y}_{p}$ is trivially an isomorphism.

If $d_{i j} \in p^{m} \mathbb{Z}-p^{m+1} \mathbb{Z}$ for some $i, j$ and some positive integer $m$, and each of every other term $d_{k \ell}$ is not in $p \mathbb{Z}$, then $L_{i}, L_{j} \in p^{m} \mathbb{Z}-p^{m+1} \mathbb{Z}$, and each of every other $L_{k}$ is not in $p \mathbb{Z}$. This shows that $\bar{Y}_{p, m}$ is a nonzero $2 \times 2$ skew-symmetric matrix (i.e., $\operatorname{det}\left(\bar{Y}_{p, m}\right) \neq 0$ ) and $M_{p, m^{\prime}}=0$ for each $m^{\prime} \neq m$. The rest follows from Lemma 4.5 and Theorem 4.6.

Next we give simple criteria for $\bar{Y}$ to be an isomorphism in the cases $n=3,4$.

Corollary 5.4. The center of $T_{q}\left[x_{1}, x_{2}, x_{3}\right]$ is a polynomial ring if and only if $\left(d_{i j}, d_{i k}\right)=1$ for all different $i, j, k$.

Proof. There are only three $d$ terms $-d_{12}, d_{13}$, and $d_{23}$. If each $\left(d_{i j}, d_{i k}\right)$ equals 1 , then no prime is a factor of more than one term in $\left\{d_{i j}\right\}$. By Corollary 5.3, the center of $T_{q}\left[x_{1}, x_{2}, x_{3}\right]$ is a polynomial ring.

Conversely, suppose that $p$ is a prime such that $d_{i j}, d_{i k} \in p \mathbb{Z}$ for some $i, j, k$. Then $L_{1}, L_{2}, L_{3} \in p \mathbb{Z}$. This implies that $M_{p}$ has dimension 3. Hence, by Corollary 5.2, $\bar{Y}_{p}$ is not an isomorphism. So $\bar{Y}$ is not an isomorphism. Therefore, the center of $T_{\boldsymbol{q}}\left[x_{1}, x_{2}, x_{3}\right]$ is not a polynomial ring by Lemma 4.3.

Corollary 5.5. The center of $T_{q}\left[x_{1}, x_{2}, x_{3}, x_{4}\right]$ is a polynomial ring if and only if, for each prime $p$, one of the following holds:

(a) $L_{i} \notin p \mathbb{Z}$ for all $i$.

(b) For some positive integer $m, \bar{Y}_{p, m}$ is $4 \times 4$ with nonzero determinant.

(c) There are distinct indices $i, j, k, \ell \in\{1,2,3,4\}$ and a nonnegative integer $m$ such that $d_{i j} \in p^{m+1} \mathbb{Z}, d_{k \ell} \in p^{m} \mathbb{Z}-p^{m+1} \mathbb{Z}$, and every other $d$ term is not in $p^{m+1} \mathbb{Z}$.

Proof. Let $P=T_{q}\left[x_{1}, x_{2}, x_{3}, x_{4}\right]$. By Lemmas 4.3 and $4.4, Z(P)$ is a polynomial ring if and only if $\bar{Y}_{p}$ is an isomorphism for all $p$. It remains to show that, for each $p, \bar{Y}_{p}$ is an isomorphism if and only if one of (a), (b), or (c) holds. Now we fix $p$ and prove the assertion in three cases according to the shape of $M_{p}$.

First we prove the "if" part.

(a) If $L_{i} \notin p \mathbb{Z}$ for all $i$, then $M_{p}=0$ and $\bar{Y}_{p}$ is trivially an isomorphism. This handles the case when $M_{p}=0$. 
(b) If for some $m>0, \bar{Y}_{p, m}$ is $4 \times 4$ with nonzero determinant, then every other $\bar{Y}_{p, r}$ (for all $r \neq m$ ) is a $0 \times 0$ matrix and, consequently, $\bar{Y}_{p}$ is an isomorphism. This is the case when $M_{p}=M_{p, m}$ is 4-dimensional for one $m$.

(c) Assume the hypotheses in part (c). Let $m^{\prime}>m$ be the integer such that $d_{i j} \in p^{m^{\prime}} \mathbb{Z}-p^{m^{\prime}+1} \mathbb{Z}$. If $m=0$, then $d_{i j}$ is the only $d$ term divisible by $p$. Hence $\bar{Y}_{p, m^{\prime}}$ is a skew-symmetric $2 \times 2$ nonzero matrix and $\bar{Y}_{p, r}$ is trivial for all $r \neq m^{\prime}$. Therefore, $\bar{Y}_{p}$ is an isomorphism. If $m>0$, then $\bar{Y}_{p, m}$ and $\bar{Y}_{p, m^{\prime}}$ are both skewsymmetric and $2 \times 2$, and (because $k_{k \ell} L_{k} / d_{k \ell} \notin p \mathbb{Z}$ ) nonzero. Furthermore, every other $\bar{Y}_{p, r}$ is $0 \times 0$ for all $r \neq m, m^{\prime}$. Therefore, $\bar{Y}_{p}$ is an isomorphism.

For the rest we prove the "only if" part.

Suppose that $\bar{Y}_{p}$ is an isomorphism. By Corollary 5.2, $M_{p}$ is even dimensional, that is, $\operatorname{dim} M_{p}=0,2$ or 4 .

The $\operatorname{dim} M_{p}=0$ case coincides with the case when $L_{i} \notin p \mathbb{Z}$ for all $i$, so we obtain case (a).

For the $\operatorname{dim} M_{p}=2$ case, at least one $d_{i j}$ lies in $p \mathbb{Z}$ and $L_{i}, L_{j}$ lie in $p \mathbb{Z}$, and no other $d$ term is a multiple of $p$, so $\bar{Y}_{p}$ is necessarily an isomorphism. We can set $m=0$, so that $d_{i j} \in p^{m+1} \mathbb{Z}$, and all other $d_{a b}$ are not in $p^{m+1} \mathbb{Z}$. So we obtain (c).

All that remains is the $\operatorname{dim} M_{p}=4$ case. Each $M_{p, m}$ is even dimensional by Corollary 5.1. If $\operatorname{dim} M_{p, m}=4$ for some $m$, then $\bar{Y}_{p, m}$ is $4 \times 4$ and $\bar{Y}_{p}$ is an isomorphism if and only if $\operatorname{det}\left(\bar{Y}_{p, m}\right) \neq 0$. So we obtain case (b).

Finally, suppose there exist $m^{\prime}>m>0$ such that $\operatorname{dim} M_{p, m}=\operatorname{dim} M_{p, m^{\prime}}=2$. Let $i, j, k, \ell$ be distinct such that $L_{i}, L_{j} \in p^{m^{\prime}} \mathbb{Z}-p^{m^{\prime}+1} \mathbb{Z}$ and $L_{k}, L_{\ell} \in p^{m} \mathbb{Z}-p^{m+1} \mathbb{Z}$. We must have that $d_{i j} \in p^{m^{\prime}} \mathbb{Z} \subseteq p^{m+1} \mathbb{Z}$ and every other $d$ term is not in $p^{m+1} \mathbb{Z}$. If $d_{k \ell} \notin p^{m} \mathbb{Z}$, then $k_{k \ell} L_{k} / d_{k \ell}, k_{\ell k} L_{\ell} / d_{\ell k} \in p \mathbb{Z}$ and $\bar{Y}_{p, m}$ is the $2 \times 2$ zero matrix, yielding a contradiction. Therefore, $d_{k \ell}$ must be in $p^{m} \mathbb{Z}$. So we obtain case (c) again.

\section{Center of generalized Weyl algebras}

Let $T$ be a commutative $k$-domain. In this section we assume that $\boldsymbol{q}:=\left\{q_{i j}\right\}$ is a set of roots of unity in $T$ and let $\mathcal{A}:=\left\{a_{i j} \mid 1 \leq i<j \leq j\right\}$ be a subset of $T$. Define the generalized Weyl algebra associated to $(\boldsymbol{q}, \mathcal{A})$ to be the central $T$-algebra

$$
V(\boldsymbol{q}, \mathcal{A}):=\frac{T\left\langle x_{1}, \ldots, x_{n}\right\rangle}{\left(x_{j} x_{i}-q_{i j} x_{i} x_{j}-a_{i j} \mid i \neq j\right)} .
$$

Consider a filtration on $V(\boldsymbol{q}, \mathcal{A})$ with $\operatorname{deg} x_{i}=1$ and $\operatorname{det} t=0$ for all $t \in T$. Suppose $\operatorname{gr} V(\boldsymbol{q}, \mathcal{A})$ is naturally isomorphic to $T_{\boldsymbol{q}}\left[x_{1}, \ldots, x_{n}\right]$.

Consider the hypothesis that,

$$
\text { for any pair }(i, j), a_{i j}=0 \text { whenever } q_{i j}=1 \text {. }
$$


Proposition 6.1. Suppose (E6.0.1) and (E6.0.2) and let $A=V(\boldsymbol{q}, \mathcal{A})$. If the center $Z(\operatorname{gr} A)$ is a polynomial ring, then so is $Z(A)$, and $Z(A) \cong Z(\operatorname{gr} A)$.

Proof. If $Z(\operatorname{gr} A)$ is a polynomial $\operatorname{ring}$, then $Z(\operatorname{gr} A)=T\left[x_{1}^{L_{1}}, \ldots, x_{n}^{L_{n}}\right]$, where $L_{i}=\operatorname{lcm}\left\{d_{i j} \mid j=1, \ldots, n\right\}$ (Lemma 4.3). Recall that $d_{i j}$ is the order of $q_{i j}$.

First we claim that $x_{i}^{L_{i}}$ is in the center of $A$. For each $j$, we have the equation $x_{j} x_{i}=q_{i j} x_{i} x_{j}+a_{i j}$. If $q_{i j}=1$, then $x_{j}$ commutes with $x_{i}$ by hypothesis (E6.0.2), so $x_{j}$ commutes with $x_{i}^{L_{i}}$. If $q_{i j} \neq 1$, then the order of $q_{i j}$ is $d_{i j}$. The equation $x_{j} x_{i}=q_{i j} x_{i} x_{j}+a_{i j}$ implies that $x_{j}$ commutes with $x_{i}^{d_{i j}}$, as each $x_{j} x_{i}^{k}$ is equal to $q_{i j}^{k} x_{i}^{k} x_{j}+\left(1+q_{i j}+\cdots+q_{i j}^{k-1}\right) a_{i j}$. Since $d_{i j}$ divides $L_{i}, x_{j}$ commutes with $x_{i}^{L_{i}}$ for all $j \neq i$. This shows that $x_{i}^{L_{i}}$ is central.

Since gr $A$ is the skew polynomial ring $T_{q}\left[x_{1}, \ldots, x_{n}\right]$, it is easy to check that $\operatorname{gr} Z(A) \subset Z(\operatorname{gr} A)$. Since $Z(\operatorname{gr} A)$ is generated by $\left\{x_{i}^{L_{i}}\right\}_{i=1}^{n}$, induction on the degree of element $f \in Z(A)$ shows that $f$ is generated by $x_{i}^{L_{i}}$. Therefore, the assertion follows.

Proposition 6.2. Retain the above notation and suppose (E6.0.1). If $a_{i j} \neq 0$ for some $i \neq j$, then $q_{i k} q_{j k}=1$ for all $k \neq i$ or $j$.

Proof. We resolve $x_{k} x_{j} x_{i}$ in two different ways:

$$
\begin{aligned}
\left(x_{k} x_{j}\right) x_{i} & =\left(q_{j k} x_{j} x_{k}+a_{j k}\right) x_{i} \\
& =q_{j k} x_{j}\left(x_{k} x_{i}\right)+a_{j k} x_{i} \\
& =q_{j k} x_{j}\left(q_{i k} x_{i} x_{k}+a_{i k}\right)+a_{j k} x_{i} \\
& =q_{j k} q_{i k}\left(x_{j} x_{i}\right) x_{k}+q_{j k} a_{i k} x_{j}+a_{j k} x_{i} \\
& =q_{j k} q_{i k}\left(q_{i j} x_{i} x_{j}+a_{i j}\right) x_{k}+q_{j k} a_{i k} x_{j}+a_{j k} x_{i} \\
& =q_{j k} q_{i k} q_{i j} x_{i} x_{j} x_{k}+q_{j k} q_{i k} a_{i j} x_{k}+q_{j k} a_{i k} x_{j}+a_{j k} x_{i},
\end{aligned}
$$

and similarly

$$
\begin{aligned}
x_{k}\left(x_{j} x_{i}\right) & =x_{k}\left(q_{i j} x_{i} x_{j}+a_{i j}\right) \\
& =q_{i j}\left(x_{k} x_{i}\right) x_{j}+a_{i j} x_{k} \\
& =q_{i j}\left(q_{i k} x_{i} x_{k}+a_{i k}\right) x_{j}+a_{i j} x_{k} \\
& =q_{i j} q_{i k} x_{i}\left(x_{k} x_{j}\right)+q_{i j} a_{i k} x_{j}+a_{i j} x_{k} \\
& =q_{i j} q_{i k} q_{j k} x_{i} x_{j} x_{k}+q_{i j} q_{i k} a_{j k} x_{i}+q_{i j} a_{i k} x_{j}+a_{i j} x_{k} .
\end{aligned}
$$

Comparing the coefficients of $x_{k}$ gives the result.

When an algebra $A$ is finitely generated and free over its center (as in the situation of Proposition 6.1), one should be able to compute the discriminant of $A$ over its center. We give an example here. 
Example 6.3. Let $A$ be generated by $x_{1}, x_{2}, x_{3}, x_{4}$ and subject to the relations

$$
\begin{aligned}
& x_{3} x_{1}-x_{1} x_{2}=0, \quad x_{4} x_{2}+x_{2} x_{4}=0, \\
& x_{3} x_{2}-x_{2} x_{3}=0, \quad x_{3} x_{4}+x_{4} x_{3}=0, \\
& x_{4} x_{1}+x_{1} x_{4}=0, \quad x_{1} x_{2}+x_{2} x_{1}=x_{3}^{2}+x_{4}^{2} .
\end{aligned}
$$

This is the example in [Vancliff and Van Rompay 2000, Lemma 1.1] (with $\lambda=0$ ). It is an iterated Ore extension, and therefore Artin-Schelter regular of global dimension 4.

It is not hard to check that the center of $A$ is generated by $x_{i}^{2}$. This algebra is a factor ring of the algebra $B$ over $T:=k[t]$ generated by $x_{1}, x_{2}, x_{3}, x_{4}$ and subject to the relations

$$
\begin{aligned}
& x_{3} x_{1}-x_{1} x_{2}=0, \quad x_{4} x_{2}+x_{2} x_{4}=0, \\
& x_{3} x_{2}-x_{2} x_{3}=0, \quad x_{3} x_{4}+x_{4} x_{3}=0 \text {, } \\
& x_{4} x_{1}+x_{1} x_{4}=0, \quad x_{1} x_{2}+x_{2} x_{1}=t .
\end{aligned}
$$

Note that $\operatorname{gr} B$ is a skew polynomial ring over $T$ with the above relations by setting $t=0$. The $Y$-matrix is

$$
\left(\begin{array}{rrrr}
0 & \frac{1}{2} & 0 & \frac{1}{2} \\
-\frac{1}{2} & 0 & 0 & \frac{1}{2} \\
0 & 0 & 0 & \frac{1}{2} \\
-\frac{1}{2} & -\frac{1}{2} & -\frac{1}{2} & 0
\end{array}\right)
$$

By Corollary 5.5(b), $B$ has center $T\left[x_{1}^{2}, x_{2}^{2}, x_{3}^{2}, x_{4}^{2}\right]$. The discriminant of $B$ over its center is $2^{48}\left(4 x_{1}^{2} x_{2}^{2}-t^{2}\right)^{8} x_{3}^{16} x_{4}^{16}$, by the next lemma. By Lemma 1.2, the discriminant of $A$ over its center is $2^{48}\left(4 x_{1}^{2} x_{2}^{2}-\left(x_{3}^{2}+x_{4}^{2}\right)^{2}\right)^{8} x_{3}^{16} x_{4}^{16}$. We will see in the next sections that $\mathbb{D}(A)=A$. As a consequence of Theorem $0.5, A$ is cancellative and the automorphism group of $A$ is affine.

Lemma 6.4. Suppose the $k[t]$-algebra $B$ is generated by $x_{1}, x_{2}, x_{3}, x_{4}$ and subject to the six relations given (E6.3.2). Then the discriminant of $B$ over its center is $2^{48}\left(4 x_{1}^{2} x_{2}^{2}-t^{2}\right)^{8} x_{3}^{16} x_{4}^{16}$.

Sketch of the proof. It is routine to check that the center of $B$ is

$$
Z(B)=k[t]\left[x_{1}^{2}, x_{2}^{2}, x_{3}^{2}, x_{4}^{2}\right] .
$$

The algebra $B$ is a free module over $Z(B)$ of rank 16 with a $Z(B)$-basis $\left\{x_{1}^{a} x_{2}^{b} x_{3}^{c} x_{4}^{d} \mid\right.$ $a, b, c, d=0,1\}$. Let $\left\{z_{1}, \ldots z_{16}\right\}$ be the above $Z(B)$-basis. Then we can compute 
the matrix $\left(\operatorname{tr}\left(z_{i} z_{j}\right)\right)_{16 \times 16}$ :

$$
\left(\begin{array}{cccccccccccccccc}
16 & 0 & 0 & 0 & 0 & 8 t & 0 & 0 & 0 & 0 & 0 & 0 & 0 & 0 & 0 & 0 \\
0 & 16 a & 8 t & 0 & 0 & 0 & 0 & 0 & 0 & 0 & 0 & 0 & 0 & 0 & 0 & 0 \\
0 & 8 t & 16 b & 0 & 0 & 0 & 0 & 0 & 0 & 0 & 0 & 0 & 0 & 0 & 0 & 0 \\
0 & 0 & 0 & 16 c & 0 & 0 & 0 & 0 & 0 & 0 & 0 & 8 c t & 0 & 0 & 0 & 0 \\
0 & 0 & 0 & 0 & 16 d & 0 & 0 & 0 & 0 & 0 & 0 & 0 & 8 d t & 0 & 0 & 0 \\
8 t & 0 & 0 & 0 & 0 & \alpha & 0 & 0 & 0 & 0 & 0 & 0 & 0 & 0 & 0 & 0 \\
0 & 0 & 0 & 0 & 0 & 0 & 16 a c & 0 & 8 c t & 0 & 0 & 0 & 0 & 0 & 0 & 0 \\
0 & 0 & 0 & 0 & 0 & 0 & 0 & -16 a d & 0 & -8 d t & 0 & 0 & 0 & 0 & 0 & 0 \\
0 & 0 & 0 & 0 & 0 & 0 & 8 c t & 0 & 16 b c & 0 & 0 & 0 & 0 & 0 & 0 & 0 \\
0 & 0 & 0 & 0 & 0 & 0 & 0 & -8 d t & 0-16 b d & 0 & 0 & 0 & 0 & 0 & 0 \\
0 & 0 & 0 & 0 & 0 & 0 & 0 & 0 & 0 & 0-16 c d & 0 & 0 & 0 & 0 & -8 c d t \\
0 & 0 & 0 & 8 c t & 0 & 0 & 0 & 0 & 0 & 0 & 0 & \beta & 0 & 0 & 0 & 0 \\
0 & 0 & 0 & 0 & 8 d t & 0 & 0 & 0 & 0 & 0 & 0 & 0 & \gamma & 0 & 0 & 0 \\
0 & 0 & 0 & 0 & 0 & 0 & 0 & 0 & 0 & 0 & 0 & 0 & 0 & 16 a c d & 8 c d t & 0 \\
0 & 0 & 0 & 0 & 0 & 0 & 0 & 0 & 0 & 0 & 0 & 0 & 0 & 8 c d t & 16 b c d & 0 \\
0 & 0 & 0 & 0 & 0 & 0 & 0 & 0 & 0 & 0 & -8 c d t & 0 & 0 & 0 & 0 & \delta
\end{array}\right)
$$

Here $\alpha=-16 a b+8 t^{2}, \beta=-16 a b c+8 c t^{2}, \gamma=-16 a b d+8 d t^{2}, \delta=16 a b c d-8 c d t^{2}$, and $a=x_{1}^{2}, b=x_{2}^{2}, c=x_{3}^{2}, d=x_{4}^{2}$. We skip the details in computing the above traces. By using Maple, its determinant is $2^{48}\left(4 x_{1}^{2} x_{2}^{2}-t^{2}\right)^{8} x_{3}^{16} x_{4}^{16}$.

\section{Three subalgebras}

In this section we discuss three (possibly different) subalgebras of $A$, all of which are helpful for the applications in the next section.

Makar-Limanov invariants. The first subalgebra is the Makar-Limanov invariant of $A$ [Makar-Limanov 1996]. This invariant has been very useful in commutative algebra. For any $k$-algebra $A$, let $\operatorname{Der}(A)$ denote the set of all $k$-derivations of $A$ and let $\operatorname{LND}(A)$ denote the set of locally nilpotent $k$-derivations of $A$.

Definition 7.1. Let $A$ be an algebra over $k$.

(1) The Makar-Limanov invariant of $A$ is

$$
\operatorname{ML}(A)=\bigcap_{\delta \in \operatorname{LND}(A)} \operatorname{ker}(\delta) .
$$

(2) We say that $A$ is $\operatorname{LND}$-rigid if $\operatorname{ML}(A)=A$, or $\operatorname{LND}(A)=\{0\}$.

(3) We say that $A$ is strongly $\operatorname{LND}$-rigid if $\operatorname{ML}\left(A\left[t_{1}, \ldots, t_{d}\right]\right)=A$ for all $d \geq 0$.

The following lemma is clear. Part (2) follows from the fact that $\partial \in \operatorname{LND}(A)$ if and only if $g^{-1} \partial g \in \operatorname{LND}(A)$. 
Lemma 7.2. Let $A$ be an algebra.

(1) $\operatorname{ML}(A)$ is a subalgebra of $A$.

(2) For any $g \in \operatorname{Aut}(A)$, we have $g(\operatorname{ML}(A))=\operatorname{ML}(A)$.

Divisor subalgebras. Throughout this subsection let $A$ be a domain containing $\mathbb{Z}$. Let $F$ be a subset of $A$. Let $\operatorname{Sw}(F)$ be the set of $g \in A$ such that $f=a g b$ for some $a, b \in A$ and $0 \neq f \in F$. Here Sw stands for "subword", which can be viewed as a divisor.

Definition 7.3. Let $F$ be a subset of $A$.

(1) Let $D_{0}(F)=F$. Inductively define $D_{n}(F)$ as the $k$-subalgebra of $A$ generated by $\operatorname{Sw}\left(D_{n-1}(F)\right)$. The subalgebra $D(F)=\bigcup_{n \geq 0} D_{n}(F)$ is called the $F$-divisor subalgebra of $A$. If $F$ is the singleton $\{f\}$, we simply write $D(\{f\})$ as $D(f)$.

(2) If $f=d(A / Z)$ (if it exists), we call $D(f)$ the discriminant-divisor subalgebra of $A$, or $D D S$ of $A$, and write it as $\mathbb{D}(A)$.

The following lemma is well-known [Makar-Limanov 2008, p. 4].

Lemma 7.4. Let $x, y$ be nonzero elements in $A$ and let $\partial \in \operatorname{LND}(A)$. If $\partial(x y)=0$, then $\partial(x)=\partial(y)=0$.

Proof. Let $m$ and $n$ be the largest integers such that $\partial^{m}(x) \neq 0$ and $\partial^{n}(y) \neq 0$. Then the product rule and the choice of $m, n$ imply that

$$
\partial^{m+n}(x y)=\sum_{i=0}^{m+n}\left(\begin{array}{c}
n+m \\
i
\end{array}\right) \partial^{i}(x) \partial^{m+n-i}(y)=\left(\begin{array}{c}
n+m \\
m
\end{array}\right) \partial^{m}(x) \partial^{n}(y) \neq 0 .
$$

So $m+n=0$. The assertion follows.

Lemma 7.5. Let $F$ be a subset of $\operatorname{ML}(A)$. Then $D(F) \subseteq \operatorname{ML}(A)$.

Proof. Let $\partial$ be any element in $\operatorname{LND}(A)$. By hypothesis, $\partial(f)=0$ for all $f \in F$. By Lemma 7.4, $\partial(x)=0$ for all $x \in \operatorname{Sw}(F)$. So $\partial=0$ when restricted to $D_{1}(F)$. By induction, $\partial=0$ when restricted to $D(F)$. The assertion follows by taking arbitrary $\partial \in \operatorname{LND}(A)$.

Lemma 7.6. Suppose $d(A / Z)$ is defined. Then the $D D S \mathbb{D}(A)$ is preserved by all $g \in \operatorname{Aut}(A)$.

Proof. By [CPWZ 2015a, Lemma 1.8(6)] or [CPWZ 2016, Lemma 1.4(4)], $d(A / Z)$ is $g$-invariant up to a unit. So, if $g \in \operatorname{Aut}(A)$, then $g$ maps $\operatorname{Sw}(d(A / Z))$ to $\operatorname{Sw}(d(A / Z))$ and $D_{1}(d(A / Z))$ to $D_{1}(d(A / Z))$. By induction, one sees that $g$ maps $D_{n}(d(A / Z))$ to $D_{n}(d(A / Z))$. So the assertion follows.

We need to find some elements $f \in A$ so that $\partial(f)=0$ for all $\partial \in \operatorname{LND}(A)$. The next lemma was proven in [CPWZ 2016, Proposition 1.5]. 
Lemma 7.7. Let $Z$ be the center of $A$ and let $d \geq 0$. Suppose $A^{\times}=k^{\times}$. Assume that $A$ is finitely generated and free over $Z$. Then we have $\partial(d(A / Z))=0$ for all $\partial \in \operatorname{LND}\left(A\left[t_{1}, \ldots, t_{d}\right]\right)$.

Proof. Let $f$ denote the element $d\left(A\left[t_{1}, \ldots, t_{d}\right] / Z\left[t_{1}, \ldots, t_{d}\right]\right)$ in $Z\left[t_{1}, \ldots, t_{d}\right]$. By [CPWZ 2016, Proposition 1.5], $\partial(f)=0$. By [CPWZ 2015a, Lemma 5.4],

$$
f={ }_{k} \times d(A / Z) .
$$

The assertion follows.

Here is the first relationship between the two subalgebras.

Proposition 7.8. Retain the hypothesis of Lemma 7.7. Let $d \geq 0$. Then

$$
\mathbb{D}(A) \subseteq \operatorname{ML}\left(A\left[t_{1}, \ldots, t_{d}\right]\right) \subseteq A .
$$

Proof. It is clear that $\operatorname{ML}\left(A\left[t_{1}, \ldots, t_{d}\right]\right) \subseteq A$ by [Bell and Zhang 2016]. Let $f$ equal $d(A / Z)$, which is in $A \subseteq A\left[t_{1}, \ldots, t_{d}\right]$. By Lemma 7.7, $f \in \operatorname{ML}\left(A\left[t_{1}, \ldots, t_{d}\right]\right)$. Let $D^{\prime}(f)$ be the discriminant-divisor subalgebra of $f$ in $A\left[t_{1}, \ldots, t_{d}\right]$. By Lemma 7.5, $D^{\prime}(f) \subseteq \operatorname{ML}\left(A\left[t_{1}, \ldots, t_{d}\right]\right)$. It is clear from the definition that $D(f) \subseteq D^{\prime}(f)$. Therefore, the assertion follows.

In particular, by taking $d=0$, we have $\mathbb{D}(A) \subseteq \operatorname{ML}(A)$.

Aut-bounded subalgebra. In this subsection we assume that $A$ is filtered such that the associated graded ring gr $A$ is a connected graded domain. Later we further assume that $A$ is connected graded. Since gr $A$ is a connected graded domain, we can define $\operatorname{deg} f$ to be the degree of gr $f$, and the degree satisfies the equation

$$
\operatorname{deg}(x y)=\operatorname{deg} x+\operatorname{deg} y
$$

for all $x, y \in A$.

Definition 7.9. Retain the above hypotheses. Let $G$ be a subgroup of $\operatorname{Aut}(A)$ and let $V$ be a subset of $A$.

(1) Let $x$ be an element in $A$. The $G$-bound of $x$ is

$$
\operatorname{deg}_{G}(x):=\sup \{\operatorname{deg}(g(x)) \mid g \in G\} .
$$

(2) Let $g$ be in $\operatorname{Aut}(A)$. The $V$-bound of $g$ is

$$
\operatorname{deg}_{g}(V):=\sup \{\operatorname{deg}(g(x)) \mid x \in V\} .
$$

(3) The G-bounded subalgebra of $A$, denoted by $\beta_{G}(A)$, is the set of elements $x$ in $A$ with finite $G$-bound. It is clear that $\beta_{G}(A)$ is a subalgebra of $A$ (Lemma 7.10(1)). In particular, the Aut-bounded subalgebra of $A$, denoted by $\beta(A)$, is the set of elements $x$ in $A$ with finite $\operatorname{Aut}(A)$-bound. 
The following lemma is easy, so we omit the proof.

Lemma 7.10. Retain the above notation. Let $G$ be a subgroup of $\operatorname{Aut}(A)$.

(1) The set $\beta_{G}(A)$ is a subalgebra of $A$.

(2) $g\left(\beta_{G}(A)\right)=\beta_{G}(A)$ for all $g \in G$.

Here is the relation between the two subalgebras $\mathbb{D}(A)$ and $\beta(A)$. Let $V$ be a subset of $A$. We say $V$ is of bounded degree if there is an $N$ such that $\operatorname{deg}(v)<N$ for all $v \in V$.

Proposition 7.11. Let $A$ be a filtered algebra such that gr $A$ is a connected graded domain. Suppose that $G \subseteq \operatorname{Aut}(A)$ and $F \subseteq A$.

(1) If $G(F)$ has bounded degree, then $D(F) \subseteq \beta_{G}(A)$.

(2) If $f \in A$ is such that $g(f)=_{Z(A) \times} f$ for all $g \in G$, then $D(f) \subseteq \beta_{G}(A)$.

(3) Assume that $A$ is finitely generated and free over its center $Z$. Let $f=d(A / Z)$. Then $\mathbb{D}(A)=D(f) \subseteq \beta(A)$.

Proof. (1) We have $D_{0}(F)=F \subseteq \beta_{G}(A)$ by assumption and use induction on $n$. Suppose that $D_{n-1}(F) \subseteq \beta_{G}(A)$. Assume that $D_{n}(F)$ is not contained in $\beta_{G}(A)$. Then there exists an $x \in D_{n}(A)$ such that $G(x)$ does not have bounded degree. Since $D_{n}(A)$ is generated by $\operatorname{Sw}\left(D_{n-1}(A)\right)$ as an algebra, there is an $f \in \operatorname{Sw}\left(D_{n-1}(A)\right)$ such that $G(f)$ does not have bounded degree. By definition of $\operatorname{Sw}\left(D_{n-1}(A)\right)$, there exists a nonzero $f^{\prime} \in D_{n-1}(A)$ and $a, b \in A$ such that $f^{\prime}=a f b$. Since gr $A$ is a domain, we have $\operatorname{deg}\left(g\left(f^{\prime}\right)\right)=\operatorname{deg}(g(a))+\operatorname{deg}(g(f))+\operatorname{deg}(g(b))$ for all $g \in G$. Hence $G\left(f^{\prime}\right)$ does not have bounded degree, which is a contradiction. Hence $D_{n}(F) \subseteq \beta_{G}(A)$ for all $n \geq 1$. Therefore, $D(F) \subseteq \beta_{G}(A)$.

(2) Since $Z(A)^{\times} \subseteq A_{0}$, we see that $G(f)$ has bounded degree, hence part (2) follows from part (1).

(3) The third assertion is a special case of part (2) by Lemma 1.2.

Under the hypotheses of Propositions 7.8 and 7.11 (and assuming that $A$ is finitely generated and free over its center $Z$ ), we have:

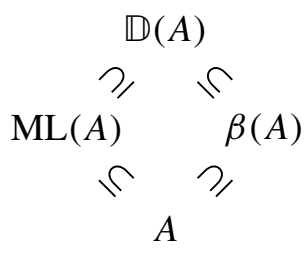

For the rest of this section, we assume that $A$ is a connected graded domain and that $k$ contains the field $\mathbb{Q}$. An automorphism $g$ of $A$ is called unipotent if

$$
g(v)=v+(\text { higher degree terms })
$$


for all homogeneous elements $v \in A$. Let $\operatorname{Aut}_{\text {uni }}(A)$ denote the subgroup of $\operatorname{Aut}(A)$ consisting of unipotent automorphisms [CPWZ 2016, after Theorem 3.1]. If $g \in \operatorname{Aut}_{\text {uni }}(A)$, we can define

$$
\log g:=-\sum_{i=1}^{\infty} \frac{1}{i}(1-g)^{i} .
$$

Let $C$ be the completion of $A$ with respect to the graded maximal ideal $\mathfrak{m}:=A_{\geq 1}$. Then $C$ is a local ring containing $A$ as a subalgebra. We can define $\operatorname{deg}_{l}: C \rightarrow \mathbb{Z}$ by setting $\operatorname{deg}_{l}(v)$ to be the lowest degree of the nonzero homogeneous components of $v \in C$. We define a unipotent automorphism of $C$ in a similar way to (E7.11.1) by using $\operatorname{deg}_{l}$. It is clear that if $g \in \operatorname{Aut}_{\text {uni }}(A)$, then it induces a unipotent automorphism of $C$, which is still denoted by $g$.

Lemma 7.12. Let $A$ be a connected graded domain. Let $g \in \operatorname{Aut}_{\mathrm{uni}}(A)$ and let $G$ be any subgroup of $\operatorname{Aut}(A)$ containing $g$. Let $B$ denote $\beta_{G}(A)$. Then $\left.(\log g)\right|_{B}$ is a locally nilpotent derivation of $B$. Further, $\left.g\right|_{B}$ is the identity if and only if $\left.(\log g)\right|_{B}$ is zero.

Proof. Let $C$ be the completion of $A$ with respect to the graded maximal ideal $\mathfrak{m}:=A_{\geq 1}$. Let $g$ also denote the algebra automorphism of $C$ induced by $g$. Then $g$ is also a unipotent automorphism of $C$.

Since $g$ is unipotent, $\operatorname{deg}_{l}(1-g)(v)>\operatorname{deg}_{l} v$ for any $0 \neq v \in C$. By induction, one has $\operatorname{deg}(1-g)^{n}(v) \geq n+\operatorname{deg} v$ for all $n \geq 1$. Thus $(\log g)(v)$ converges and therefore is well-defined. It follows from a standard argument that $\log g$ is a derivation of $C$ (this is also a consequence of [Freudenburg 2006, Proposition 2.17(b)]).

Let $v$ be an element in $B:=\beta_{G}(A)$. Note that $g^{n}(v) \in B$ for all $n$ by Lemma 7.10. Since $v \in B$, there is an $N_{0}$ such that $\operatorname{deg} g^{n}(v)<N_{0}$ for all $n$. If $(1-g)^{n}(v) \neq 0$, then

$$
\operatorname{deg}(1-g)^{n}(v)=\operatorname{deg}\left(\sum_{i=0}^{n}\left(\begin{array}{c}
n \\
i
\end{array}\right) g^{i}(v)\right)<N_{0} \quad \text { for all } n .
$$

When $n \geq N_{0}$, the inequalities from the previous paragraph imply that

$$
\operatorname{deg}_{l}(1-g)^{n}(v) \geq n+\operatorname{deg} v \geq N_{0},
$$

which contradicts $(\mathrm{E} 7.12 .1)$ unless $(1-g)^{n}(v)=0$. Therefore,

$$
(1-g)^{n}(v)=0 \quad \text { for all } n>N_{0} .
$$

By (E7.12.3), the infinite sum of $\log g$ in (E7.11.2) terminates when applied to $v \in B$, and $(\log g)(v) \in A$. By Lemma 7.10, $(\log g)(v) \in B$. Since $\log g$ is a derivation of $C$, it is a derivation when restricted to $B$. 
Next we need to show that it is a locally nilpotent derivation when restricted to $B$. It suffices to verify that, for any $v \in B,(\log g)^{N}(v)=0$ for $N \gg 0$, which follows from (E7.11.2) and (E7.12.3).

The final assertion follows from the fact that $g$ is the exponential function of $\log g$ and $\log g$ is locally nilpotent.

Now we are ready to prove the second part of Theorem 0.5 without the finite GK-dimension hypothesis.

Theorem 7.13. Let $k$ be a field of characteristic zero and let $A$ be a connected graded domain over $k$. Assume that A is finitely generated and free over its center $Z$ in part (2).

(1) If $\operatorname{ML}(A)=\beta(A)=A$, then $\operatorname{Aut}_{u n i}(A)=\{1\}$.

(2) If $\mathbb{D}(A)=A$, then $\operatorname{Aut}_{\mathrm{uni}}(A)=\{1\}$.

Proof. (1) By hypothesis, $B:=\beta(A)$ equals $A$. Let $g \in$ Aut $_{\text {uni }}(A)$. Then $\left.(\log g)\right|_{B}$ is a locally nilpotent derivation of $B$ by Lemma 7.12. Hence $\log g \in \operatorname{LND}(A)$. Since $\operatorname{ML}(A)=A$, we have $\operatorname{LND}(A)=\{0\}$. So $\log g=0$. By Lemma 7.12, $g$ is the identity.

(2) Combining the hypothesis $\mathbb{D}(A)=A$ with Propositions 7.8 and 7.11, we have $\operatorname{ML}(A)=\beta(A)=A$. The assertion follows from part (1).

\section{Applications}

In this section we assume that $k$ is a field of characteristic zero.

Zariski cancellation problem. The Zariski cancellation problem for noncommutative algebras was studied in [Bell and Zhang 2016]. We recall some definitions and results.

Definition 8.1. [Bell and Zhang 2016, Definition 1.1] Let $A$ be an algebra.

(1) We call $A$ cancellative if $A[t] \cong B[t]$ for some algebra $B$ implies that $A \cong B$.

(2) We call $A$ strongly cancellative if, for any $d \geq 1, A\left[t_{1}, \ldots, t_{d}\right] \cong B\left[t_{1}, \ldots, t_{d}\right]$ for some algebra $B$ implies that $A \cong B$.

The original Zariski cancellation problem, or ZCP, asks if the polynomial ring $k\left[t_{1}, \ldots, t_{n}\right]$, where $k$ is a field, is cancellative. A recent result of Gupta [2014a; 2014b] settled the question negatively in positive characteristic for $n \geq 3$. The ZCP in characteristic zero remains open for $n \geq 3$. Some history and partial results can be found in [Bell and Zhang 2016], where the authors used discriminants and locally nilpotent derivations to study the ZCP for noncommutative rings.

One of their main results is the following. 
Theorem 8.2 [Bell and Zhang 2016, Theorems 0.4 and 3.3]. Let A be a finitely generated domain of finite Gelfand-Kirillov dimension. If A is strongly LND-rigid (respectively, LND-rigid), then A is strongly cancellative (respectively, cancellative).

Now we have an immediate consequence, which is the first part of Theorem 0.5. Combining it with Theorem 7.13, we have finished the proof of Theorem 0.5.

Theorem 8.3. Let $A$ be a finitely generated domain of finite $G K$-dimension. Let $Z$ be the center of $A$ and suppose $A^{\times}=k^{\times}$. Assume that $A$ is finitely generated and free over $Z$. If $A=\mathbb{D}(A)$, then $A$ is strongly cancellative.

Proof. Combining the hypothesis $A=\mathbb{D}(A)$ with Proposition 7.8, we have

$$
A=\mathbb{D}(A) \subseteq \operatorname{ML}\left(A\left[t_{1}, \ldots, t_{d}\right]\right) \subseteq A .
$$

So $\operatorname{ML}\left(A\left[t_{1}, \ldots, t_{d}\right]\right)=A$, or $A$ is strongly LND-rigid. The assertion follows from Theorem 8.2.

Next we give two examples.

Example 8.4. Let $A$ be generated by $x_{1}, x_{2}, x_{3}, x_{4}$ and subject to the relations

$$
\begin{array}{ll}
x_{1} x_{2}+x_{2} x_{1}=0, & x_{2} x_{3}+x_{3} x_{2}=0, \\
x_{1} x_{3}+x_{3} x_{1}=0, & x_{3} x_{4}+x_{4} x_{3}=0, \\
x_{1} x_{4}+x_{4} x_{1}=x_{3}^{2}, & x_{2} x_{4}+x_{4} x_{2}=0 .
\end{array}
$$

This is an iterated Ore extension, so it is Artin-Schelter regular of global dimension 4. This is a special case of the algebra in [Vancliff et al. 1998, Definition 3.1]. Set $x_{i}^{2}=y_{i}$ for $i=1, \ldots, 4$. Then $Z(A)=k\left[y_{1}, y_{2}, y_{3}, y_{4}\right]$. The $M_{1}$-matrix of (E3.0.1) is

$$
\left(a_{i j}\right)_{4 \times 4}=\left(\begin{array}{cccc}
2 y_{1} & 0 & 0 & y_{3} \\
0 & 2 y_{2} & 0 & 0 \\
0 & 0 & 2 y_{3} & 0 \\
y_{3} & 0 & 0 & 2 y_{4}
\end{array}\right) .
$$

The determinant $\operatorname{det}\left(a_{i j}\right)$ is $f_{0}:=4 y_{2} y_{3}\left(4 y_{1} y_{4}-y_{3}^{2}\right)$. By Theorem 3.7, the discriminant $f:=d(A / Z)$ is $f_{0}^{2^{3}}$. It is clear that $y_{2}, y_{3} \in \operatorname{Sw}(f)$ and $y_{1}, y_{4} \in \operatorname{Sw}\left(D_{1}(f)\right)$. Thus $x_{i} \in \operatorname{Sw}\left(D_{2}(f)\right)$ for all $i$. Consequently, $A=\mathbb{D}(A)$. By Theorem 8.3, $A$ is strongly cancellative.

The next example is somewhat generic.

Example 8.5. Let $T$ be a commutative domain and let $A=C(V, q)$ be the Clifford algebra associated to a quadratic form $q: V \rightarrow T$ where $V$ is a free $T$-module of rank $n$. Suppose that $n$ is even. Then the center of $A$ is $T$ [Lam 2005, Chapter 5, Theorem 2.5(a)]. We assume that $A$ is a domain with $A^{\times}=k^{\times}$. Let $t_{1}, \ldots, t_{w}$ be a set of generators of $T$, and suppose that $q(V) \subseteq\left(t_{1} \cdots t_{w}\right) T$ or $\operatorname{det}(q) \in\left(t_{1} \cdots t_{w}\right) T$. 
Then by Theorem 3.7 we have $f:=d(A / T) \in\left(t_{1} \cdots t_{w}\right)^{2^{n-1}}$. So $t_{s} \in \operatorname{Sw}(f)$ for all $s$. This shows that $T \subseteq \mathbb{D}(A)$ and then $A=\mathbb{D}(A)\left(\right.$ as $\left.x_{i}^{2} \in T\right)$. By Theorem 8.3, $A$ is strongly cancellative.

Remark 8.6. Let $A$ be the algebra in Example 6.3. Using the formula for $d(A / Z)$ given in Lemma 6.4 , it is easy to see that $A=\mathbb{D}(A)$. So $A$ is cancellative by Theorem 8.3.

Automorphism problem. By [CPWZ 2015a; 2016], the discriminant controls the automorphism group of some noncommutative algebras. In this section we compute some automorphism groups by using the discriminants computed in previous sections. We first recall some definitions and results.

We modify the definitions in [CPWZ 2015a; 2016] slightly. Let $A$ be an $\mathbb{N}$-filtered algebra such that gr $A$ is a connected graded domain. Let $X:=\left\{x_{1}, \ldots, x_{n}\right\}$ be a set of elements in $A$ such that it generates $A$ and gr $X$ generates gr $A$. We $\operatorname{donot}$ require $\operatorname{deg} x_{i}=1$ for all $i$.

Definition 8.7. Let $f$ be an element in $A$ and let $X^{\prime}=\left\{x_{1}, \ldots, x_{m}\right\}$ be a subset of $X$. We say $f$ is dominating over $X^{\prime}$ if, for any subset $\left\{y_{1}, \ldots, y_{n}\right\} \subseteq A$ that is linearly independent in the quotient $k$-space $A / k$, there is a lift of $f$, say $F\left(X_{1}, \ldots, X_{n}\right)$, in the free algebra $k\left\langle X_{1}, \ldots, X_{n}\right\rangle$, such that $\operatorname{deg} F\left(y_{1}, \ldots, y_{n}\right)>\operatorname{deg} f$ whenever $\operatorname{deg} y_{i}>\operatorname{deg} x_{i}$ for some $x_{i} \in X^{\prime}$.

The following lemma is easy.

Lemma 8.8. Retain the above notation. Suppose $f:=d(A / Z)$ is dominating over $X^{\prime}$. Then for every automorphism $g \in \operatorname{Aut}(A)$, we have $\operatorname{deg} g\left(x_{i}\right) \leq \operatorname{deg} x_{i}$ for all $x_{i} \in X^{\prime}$.

Proof. Let $y_{i}=g\left(x_{i}\right)$. Then $\left\{y_{1}, \ldots, y_{n}\right\}$ is linearly independent in $A / k$ (as $\left\{x_{1}, \ldots, x_{n}\right\}$ is linearly independent in $\left.A / k\right)$. If $\operatorname{deg} y_{i}>\operatorname{deg} x_{i}$ for some $i$, by the dominating property, there is a lift of $f$ in the free algebra, say $F\left(X_{1}, \ldots, X_{n}\right)$, such that $\operatorname{deg} F\left(y_{1}, \ldots, y_{n}\right)>\operatorname{deg} f$. Since $g$ is an algebra automorphism,

$$
F\left(y_{1}, \ldots, y_{n}\right)=F\left(g\left(x_{1}\right), \ldots, g\left(x_{n}\right)\right)=g\left(F\left(x_{1}, \ldots, x_{n}\right)\right)=g(f) .
$$

By [CPWZ 2015a, Lemma 1.8(6)], $g(f)=f$ (up to a unit in $Z$ ). Hence

$$
\operatorname{deg} F\left(y_{1}, \ldots, y_{n}\right)=\operatorname{deg} g(f)=\operatorname{deg} f,
$$

yielding a contradiction. Therefore, $\operatorname{deg} g\left(x_{i}\right)=\operatorname{deg} y_{i} \leq \operatorname{deg} x_{i}$ for all $i$.

We will study the automorphism group of a class of Clifford algebras; see Example 8.5.

Example 8.9. Let $A$ be the Clifford algebra over a commutative $k$-domain $T$ as in Example 8.5 and assume that $n$ is even. Let $\left\{z_{1}, \ldots, z_{n}\right\}$ denote a set of generators 
for $A$. We will use $\left\{x_{1}, \ldots, x_{n}\right\}$ for the generators of the generic Clifford algebra $A_{g}$ defined in Section 3. Then there is an algebra homomorphism from $A_{g}$ to $A$ sending $x_{i}$ to $z_{i}$ for all $i$. Since $n$ is even, $T$ is the center of $A$. Assume that $A$ is a filtered algebra such that $\operatorname{gr} A$ is a connected graded domain, so we can define the degree of any nonzero element in $A$. Further assume that $\operatorname{deg} t_{i}=2$ (not 1$)$ for all $i=1, \ldots, w$ and $\operatorname{deg} z_{i}>2$ for all $i=1,2, \ldots, n$. In particular, there is no element of degree 1 . Some explicit examples are given later in this example.

Recall that we assumed $q(V) \subseteq\left(t_{1} \cdots t_{w}\right) T$. Let $2 b_{i j}=z_{j} z_{i}+z_{i} z_{j}$. Then we can write $b_{i j}=\left(t_{1} \cdots t_{w}\right)^{N} b_{i j}^{\prime}$ for some $N>0$. By Theorem 3.7, the discriminant is $f:=d(A / T)=\left[\left(\prod_{s=1}^{w} t_{s}\right)^{N} d^{\prime}\right]^{2^{n-1}}$, where $d^{\prime}=\operatorname{det}\left(2 b_{i j}^{\prime}\right)_{n \times n}$. We need another hypothesis, which is that

$$
\operatorname{deg} d^{\prime}<N
$$

Let $X^{\prime}=\left\{t_{i}\right\}_{i=1}^{w}$ and $X=\left\{z_{i}\right\}_{i=1}^{n} \cup X^{\prime}$. Then $f$ is a noncommutative polynomial over $X^{\prime}$. We first claim that $f$ is dominating over $X^{\prime}$. Let $\left\{y_{i}\right\}_{i=1}^{w}$ be a set of elements in $A \backslash k$. If $\operatorname{deg} y_{i}>2$ for some $i$, then $\operatorname{deg}\left[\left(\prod_{s=1}^{w} y_{s}\right)^{N} d^{\prime}\left(y_{1}, \ldots, y_{w}\right)\right]^{2 n-1}$ is strictly larger than the degree of $f$, as we assume that $\operatorname{deg} d^{\prime}<N$. This shows the claim.

Now let $g$ be any algebra automorphism of $A$ and let $y_{i}$ be $g\left(t_{i}\right)$ for all $i$. Then, by Lemma 8.8, $\operatorname{deg} y_{i}=2$. It follows from the relations $z_{i} z_{i}=b_{i i}$ that $\operatorname{deg} z_{i}>3$. Hence $(\operatorname{gr} A)_{2}$ is generated by the $t_{i}$. This implies that $y_{i}$ is in the span of $X^{\prime}$ and $k$. In some sense, every automorphism of $A$ is affine (with respect to $X^{\prime}$ ). It is a big step in understanding the automorphism group of $A$.

Below we study the automorphism group of a family of subalgebras of the generic Clifford algebra $A_{g}$ of rank $n$ that is defined in Section 3. As before, we assume $n$ is even. We have two different sets of variables $t$, one for $A_{g}$ and the other for general $A$. It would be convenient to unify these in the following discussion. So we identify $\left\{t_{(i, j)} \mid 1 \leq i \leq j \leq n\right\}$ with $\left\{t_{i}\right\}_{i=1}^{w}$ via a bijection $\phi$. Here $w=\frac{1}{2} n(n+1)$ as in the definition of $A_{g}$ (Section 3).

Let $r$ be any positive integer and let $B_{g, r}$ be the graded subalgebra of $A_{g}$ generated by $\left\{t_{(i, j)}\right\}$ for all $1 \leq i \leq j \leq n$ (or $\left.\left\{t_{i}\right\}_{i=1}^{w}\right)$ and $z_{i}:=x_{i}\left(\prod_{k=1}^{w} t_{k}\right)^{r}$ for all $i=1,2, \ldots, n$. Since $B_{g, r}$ is a graded subalgebra of $A_{g}$, it is a connected graded domain. This is also a Clifford algebra over $T_{g}:=k\left[t_{(i, j)}\right]$ generated by $z_{1}, \ldots, z_{n}$ and subject to the relations

$$
z_{j} z_{i}+z_{i} z_{j}=2\left(\prod_{k=1}^{w} t_{k}\right)^{2 r} t_{(i, j)}=: 2 b_{i j}
$$

from which the bilinear form $b$ and associated quadratic form $q$ can easily be recovered. In particular, $q(V) \subseteq\left(\prod_{k=1}^{w} t_{k}\right)^{2 r} T_{g}$, where $V=\bigoplus_{i=1}^{n} T_{g} z_{i}$. By the definition of $A_{g}$, we have $\operatorname{deg} t_{i}=2$. Then $\operatorname{deg} z_{i}=1+4 r w>3$. Now we assume 
that $N:=2 r$ is bigger than $2 n$, which is the degree of $d^{\prime}:=\operatorname{det}\left(t_{(i, j)}\right)$. So we have

$$
n<r, \quad \text { or equivalently } \operatorname{deg} d^{\prime}<N,
$$

as required by (E8.9.1). See also Remark 8.10.

Let $g$ be an algebra automorphism of $B_{g, d}$. By the above discussion, $g\left(t_{i}\right)$, for each $i$, is a linear combination of $\left\{t_{j}\right\}_{j=1}^{w}$ and 1 . Using the relations $z_{i}^{2}=b_{i i}$, we see that $\operatorname{deg} g\left(z_{i}\right)=\operatorname{deg}\left(z_{i}\right)$ for all $i$. Thus $g$ must be a filtered automorphism of $B_{g, d}$.

Since $g$ preserves the discriminant $f$ and $f$ is homogeneous in $t_{i}$, we have $\operatorname{deg} g\left(t_{i}\right)=2$. Further, by using the expression of $f$ and the fact that $T_{g}$ is a UFD, $g\left(t_{i}\right)$ can not be a linear combination of the $t_{j}$ of more than one term. Thus $g\left(t_{i}\right)=c_{i} t_{j}$ for some $j$ and some $c_{i} \in k^{\times}$. This implies that there is a permutation $\sigma \in S_{w}$ and a collection of units $\left\{c_{i}\right\}_{i=1}^{w}$ such that $g\left(t_{i}\right)=c_{i} t_{\sigma(i)}$ for all $i$. Since $g$ is filtered (by the last paragraph), $g\left(z_{i}\right)=\sum_{h=1}^{n} d_{i h} z_{h}+e_{i}$, where $d_{i h}, e_{i} \in k$. Applying $g$ to the relation

$$
z_{i}^{2}=b_{i i}=\left(\prod_{i=1}^{w} t_{i}\right)^{N} t_{\phi(i, i)}, \quad \text { where } N:=2 r,
$$

we obtain that

$$
\left(\sum_{h} d_{i h} z_{h}\right)^{2}+2 e_{i}\left(\sum_{h} d_{i h} z_{h}\right)+e_{i}^{2}=\left(\prod_{i=1}^{w} c_{i} t_{i}\right)^{N} g\left(t_{\phi(i, i)}\right) .
$$

Since $\left(\sum_{h} d_{i h} z_{h}\right)^{2} \in T$, we have $e_{i}\left(\sum_{h} d_{i h} z_{h}\right)=0$. Consequently, $e_{i}=0$ and $g\left(z_{i}\right)=\sum_{h=1}^{n} d_{i h} z_{h}$. Applying $g$ to the relations

$$
z_{i} z_{j}+z_{j} z_{i}=2 b_{i j}=2\left(\prod_{i=1}^{w} t_{i}\right)^{N} t_{\phi(i, j)}
$$

and expanding the left-hand side, we obtain

$$
\sum_{h, l} d_{i h} d_{j l}\left(z_{h} z_{l}+z_{l} z_{h}\right)=2\left(\prod_{i=1}^{w} c_{i} t_{i}\right)^{N} g\left(t_{\phi(i, j)}\right) .
$$

Hence $d_{i h} d_{j l}$ is nonzero for only one pair $(h, l)$. Thus there is a set of units $\left\{d_{i}\right\}_{i=1}^{n}$ and a permutation $\psi \in S_{n}$ such that $g\left(z_{i}\right)=d_{i} z_{\psi(i)}$ for all $i=1, \ldots, n$. Then the above equation implies that

$$
d_{i} d_{j}\left(\prod_{i=1}^{w} t_{i}\right)^{N} t_{\phi(\psi(i), \psi(j))}=\left(\prod_{i=1}^{w} c_{i}\right)^{N}\left(\prod_{i=1}^{w} t_{i}\right)^{N} c_{\phi(i, j)} t_{\sigma(\phi(i, j))}
$$

for all $i, j$. Therefore,

$$
\phi(\psi(i), \psi(j))=\sigma(\phi(i, j))
$$


and

$$
d_{i} d_{j}=\left(\prod_{i=1}^{w} c_{i}\right)^{N} c_{\phi(i, j)}
$$

for all $i, j$.

By (E8.9.2), $\sigma$ is completely determined by $\psi \in S_{n}$. Let $\bar{d}_{i}=d_{i}\left(\prod_{i=1}^{w} c_{i}\right)^{-r}$. Then (E8.9.3) says that $\bar{d}_{i} \bar{d}_{j}=c_{\phi(i, j)}$. So $\prod_{i=1}^{w} c_{i}=\prod_{1 \leq i \leq j \leq n} \bar{d}_{i} \bar{d}_{j}$. This means the $c_{\phi(i, j)}$ and $d_{i}$ are completely determined by the $\bar{d}_{i}$. In conclusion,

$$
\operatorname{Aut}\left(B_{g, r}\right) \cong\left\{\psi \in S_{n}\right\} \ltimes\left\{\bar{d}_{i} \in k^{\times} \mid i=1, \ldots, n\right\} \cong S_{n} \ltimes\left(k^{\times}\right)^{n} .
$$

In particular, every algebra automorphism of $B_{g, r}$ is a graded algebra automorphism.

Remark 8.10. As a consequence of the computation in Example 8.9, $\operatorname{Aut}\left(B_{g, r}\right)$ is independent of the parameter $r$ when $r>n$. In fact, this assertion holds for all $r>0$, but its proof requires a different and longer analysis, so it is omitted. On the other hand, $\operatorname{Aut}\left(B_{g, 0}\right)=\operatorname{Aut}\left(A_{g}\right)$ is very different; see Remark 3.9(3).

We will work out one more automorphism group below.

Example 8.11. We continue to study Example 8.4 and prove that every algebra automorphism of $A$ in Example 8.4 is graded. Some unimportant details are omitted due to the length.

Claim 1: $\mathfrak{m}:=A_{\geq 1}$ is the only ideal of codimension 1 satisfying $\operatorname{dim} \mathfrak{m} / \mathfrak{m}^{2}=4$. Suppose $I=\left(x_{1}-a_{1}, x_{2}-a_{2}, x_{3}-a_{3}, x_{4}-a_{4}\right)$ is an ideal of $A$ of codimension 1 such that $\operatorname{dim}_{k} I / I^{2}=4$. Then the map $\pi: x_{i} \rightarrow a_{i}$ for all $i$ extends to an algebra homomorphism $A \rightarrow k$. Applying $\pi$ to the relations of $A$ in (E8.4.1), we obtain

$$
a_{1} a_{2}=0, \quad a_{1} a_{3}=0, \quad 2 a_{1} a_{4}=a_{3}^{2}, \quad a_{2} a_{3}=0, \quad a_{3} a_{4}=0, \quad a_{2} a_{4}=0 .
$$

Therefore, $\left(a_{i}\right)$ is either $\left(a_{1}, 0,0,0\right)$, or $\left(0, a_{2}, 0,0\right)$, or $\left(0,0,0, a_{4}\right)$. By symmetry, we consider the first case and the details of the other cases are omitted. Let $z_{i}=x_{i}-a_{i}$ for all $i$. Then the first relation of (E8.4.1) becomes

$$
z_{1} z_{2}+z_{2} z_{1}=\left(x_{1}-a_{1}\right) x_{2}+x_{2}\left(x_{1}-a_{1}\right)=-2 a_{1} x_{2}=-2 a_{1} z_{2} .
$$

So $2 a_{1} z_{2} \in I^{2}$. Since $\operatorname{dim} I / I^{2}=4$, we have $a_{1}=0$. Thus we have proved Claim 1 .

One of the consequences of Claim 1 is that any algebra automorphism of $A$ preserves $\mathfrak{m}$. So we have a short exact sequence

$$
1 \rightarrow \operatorname{Aut}_{\mathrm{uni}}(A) \rightarrow \operatorname{Aut}(A) \rightarrow \operatorname{Aut}_{\mathrm{gr}}(A) \rightarrow 1,
$$

where $\operatorname{Aut}_{\mathrm{gr}}(A)$ is the group of graded algebra automorphisms of $A$ and $\operatorname{Aut}_{\mathrm{uni}}(A)$ is the group of unipotent algebra automorphisms of $A$.

Claim 2: If $f$ is a nonzero normal element in degree 1 , then $B:=A /(f)$ is an Artin-Schelter regular domain of global dimension 3. By [Rogalski and Zhang 2012, 
Lemma 1.1], $B$ has global dimension 3. Since $A$ satisfies the $\chi$-condition [Artin and Zhang 1994], so does $B$. As a consequence, $B$ is AS regular of global dimension 3 [Artin and Schelter 1987]. It is well-known that every Artin-Schelter regular algebra of global dimension 3 is a domain (following by the Artin-Schelter-TateVan den Bergh classification [Artin and Schelter 1987; Artin et al. 1991; 1990]).

Claim 3: If $f \in A_{1}$ is a normal element, then $f \in k x_{2}$ or $f \in k x_{3}$. First of all, both $x_{2}$ and $x_{3}$ are normal elements by the relations (E8.4.1). Note that $x_{i} g=\eta_{-1}(g) x_{i}$ for $i=2,3$, where $\eta_{-1}$ is the algebra automorphism of $A$ sending $x_{i}$ to $-x_{i}$ for all $i$.

Suppose that $f$ is nonzero normal and $f \notin k x_{3} \cup k x_{4}$. Then the image $\bar{f}$ of $f$ is normal in $A /\left(x_{3}\right)$. Since $A /\left(x_{3}\right)$ is a skew polynomial ring, by [Kirkman et al. 2010, Lemma 3.5(d)], $\bar{f}$ is a scalar multiple of $x_{i}$ for some $i=1,2$, or 4 . This implies that $f$ is either $a x_{1}+b x_{3}$, or $a x_{2}+b x_{3}$, or $a x_{4}+b x_{3}$ for some $a, b \in k$. If $b=0$, then $f=x_{1}$ or $x_{4}$. The relation $x_{1} x_{4}+x_{4} x_{1}=x_{3}^{2}$ implies that $A /(f)$ is not a domain (as $x_{3}^{2}=0$ in $A /(f)$ ). This contradicts Claim 2. So the only possible case is $f=x_{2}$ (again yielding a contradiction). Now assume that $b \neq 0$ (and $a \neq 0$ because $\left.f \notin k x_{3} \cup k x_{4}\right)$. We consider the first case and the details of the other cases are similar and omitted. Since $f=a x_{1}+b x_{3}$, the relation $x_{1} x_{3}+x_{3} x_{1}=0$ implies that $x_{1}^{2}=0$ in $A /(f)$, which contradicts Claim 2. In all these cases, we obtain a contradiction, and therefore $f \in k x_{2}$ or $f \in k x_{3}$.

Since $A /\left(x_{2}\right)$ is not isomorphic to $A /\left(x_{3}\right)$, there is no algebra automorphism sending $x_{2}$ to $x_{3}$. As a consequence, any graded automorphism $\psi$ of $A$ maps $x_{2} \rightarrow c_{2} x_{2}$ and $x_{3} \rightarrow c_{3} x_{3}$. Let $g$ be any graded algebra automorphism of $A$. Let $\bar{g}$ be the induced algebra automorphism of $A /\left(x_{3}\right)$. By [Kirkman et al. 2010, Lemma 3.5(e)], $\bar{g}$ sends $x_{1} \rightarrow c_{1} x_{1}$ and $x_{4} \rightarrow c_{4} x_{4}$, or $x_{1} \rightarrow c_{1} x_{4}$ and $x_{4} \rightarrow c_{4} x_{1}$. Then, by using the original relations in (E8.4.1), one can check that $g$ is of the form

$$
x_{1} \rightarrow c_{1} x_{1}, \quad x_{2} \rightarrow c_{2} x_{2}, \quad x_{3} \rightarrow c_{3} x_{3}, \quad x_{4} \rightarrow c_{4} x_{4},
$$

where $c_{1} c_{2}=c_{3}^{2}=c_{4}^{2}$, or

$$
x_{1} \rightarrow c_{1} x_{4}, \quad x_{2} \rightarrow c_{2} x_{2}, \quad x_{3} \rightarrow c_{3} x_{3}, \quad x_{4} \rightarrow c_{4} x_{1},
$$

where $c_{1} c_{2}=c_{3}^{2}=c_{4}^{2}$. So

$$
\operatorname{Aut}_{\mathrm{gr}}(A) \cong\left\{\left(c_{1}, c_{2}, c_{3}, c_{4}\right) \in\left(k^{\times}\right)^{4} \mid c_{1} c_{2}=c_{3}^{2}=c_{4}^{2}\right\},
$$

which is completely determined.

Claim 4: $\operatorname{Aut}_{\text {uni }}(A)$ is trivial. Recall that the discriminant of $A$ over its center is

$$
d:=\left(x_{2}^{2} x_{3}^{2}\left(4 x_{1}^{2} x_{4}^{2}-x_{3}^{4}\right)\right)^{8} .
$$

By Example 8.4, the DDS subalgebra $\mathbb{D}(A)$ is the whole algebra $A$. The assertion follows from Theorem 0.5 . 
Combining all these claims, one sees that $\operatorname{Aut}(A)=\operatorname{Autgr}_{\mathrm{gr}}(A)$, which is described in Claim 3.

Remark 8.12. Ideas as in Remark 8.10 also apply to Example 6.3 and a similar conclusion holds. The interested reader can fill out the details.

\section{Acknowledgements}

The authors would like to thank the referees for their careful reading and valuable comments. A. A. Young was supported by the US National Science Foundation (NSF Postdoctoral Research Fellowship, No. DMS-1203744) and J. J. Zhang was supported by the US National Science Foundation (Nos. DMS-0855743 and DMS1402863).

\section{References}

[Artin and Schelter 1987] M. Artin and W. F. Schelter, "Graded algebras of global dimension 3", Adv. Math. 66:2 (1987), 171-216. MR 917738 Zbl 0633.16001

[Artin and Zhang 1994] M. Artin and J. J. Zhang, "Noncommutative projective schemes", Adv. Math. 109:2 (1994), 228-287. MR 1304753 Zbl 0833.14002

[Artin et al. 1990] M. Artin, J. Tate, and M. Van den Bergh, "Some algebras associated to automorphisms of elliptic curves", pp. 33-85 in The Grothendieck Festschrift, vol. 1, edited by P. Cartier et al., Progress in Mathematics 86, Birkhäuser, Boston, MA, 1990. MR 1086882 Zbl 0744.14024

[Artin et al. 1991] M. Artin, J. Tate, and M. Van den Bergh, "Modules over regular algebras of dimension 3", Invent. Math. 106:2 (1991), 335-388. MR 1128218 Zbl 0763.14001

[Bell and Zhang 2016] J. Bell and J. J. Zhang, "Zariski cancellation problem for noncommutative algebras", preprint, 2016. arXiv 1601.04625

[CPWZ 2015a] S. Ceken, J. H. Palmieri, Y.-H. Wang, and J. J. Zhang, “The discriminant controls automorphism groups of noncommutative algebras", Adv. Math. 269 (2015), 551-584. MR 3281142 Zbl 06374153

[CPWZ 2015b] S. Ceken, J. H. Palmieri, Y.-H. Wang, and J. J. Zhang, "Invariant theory for quantum Weyl algebras under finite group action”, preprint, 2015. arXiv 1501.07881

[CPWZ 2016] S. Ceken, J. H. Palmieri, Y.-H. Wang, and J. J. Zhang, "The discriminant criterion and automorphism groups of quantized algebras", Adv. Math. 286 (2016), 754-801. MR 3415697 Zbl 06506332

[Freudenburg 2006] G. Freudenburg, Algebraic theory of locally nilpotent derivations, Encyclopaedia of Mathematical Sciences 136, Springer, Berlin, 2006. MR 2259515 Zbl 1121.13002

[Gupta 2014a] N. Gupta, "On the cancellation problem for the affine space $\mathbb{A}^{3}$ in characteristic $p$ ", Invent. Math. 195:1 (2014), 279-288. MR 3148104 Zbl 1309.14050

[Gupta 2014b] N. Gupta, "On Zariski's cancellation problem in positive characteristic", Adv. Math. 264 (2014), 296-307. MR 3250286 Zbl 1325.14078

[Kirkman et al. 2010] E. Kirkman, J. Kuzmanovich, and J. J. Zhang, "Shephard-Todd-Chevalley theorem for skew polynomial rings", Algebr. Represent. Theory 13:2 (2010), 127-158. MR 2601538 Zbl 1215.16032 
[Lam 2005] T. Y. Lam, Introduction to quadratic forms over fields, Graduate Studies in Mathematics 67, American Mathematical Society, Providence, RI, 2005. MR 2104929 Zbl 1068.11023

[Levasseur 1992] T. Levasseur, "Some properties of noncommutative regular graded rings", Glasgow Math. J. 34:3 (1992), 277-300. MR 1181768 Zbl 0824.16032

[Lu et al. 2007] D.-M. Lu, J. H. Palmieri, Q.-S. Wu, and J. J. Zhang, "Regular algebras of dimension 4 and their $A_{\infty}$-Ext-algebras", Duke Math. J. 137:3 (2007), 537-584. MR 2309153 Zbl 1193.16014

[Makar-Limanov 1996] L. Makar-Limanov, "On the hypersurface $x+x^{2} y+z^{2}+t^{3}=0$ in $\mathbb{C}^{4}$ or a $\mathbb{C}^{3}$-like threefold which is not $\mathbb{C}^{3}$ ", Israel J. Math. 96:2 (1996), 419-429. MR 1433698 Zbl 0896.14021

[Makar-Limanov 2008] L. Makar-Limanov, "Locally nilpotent derivations, a new ring invariant and applications", preprint, 2008, available at http://www.math.wayne.edu/ lml/lmlnotes.pdf.

[Reiner 1975] I. Reiner, Maximal orders, London Mathematical Society Monographs 5, Academic Press, London, 1975. Reprinted by Oxford University Press, 2003. MR 0393100 Zbl 0305.16001

[Rogalski and Zhang 2012] D. Rogalski and J. J. Zhang, "Regular algebras of dimension 4 with 3 generators", pp. 221-241 in New trends in noncommutative algebra, edited by P. Ara et al., Contemporary Mathematics 562, American Mathematical Society, Providence, RI, 2012. MR 2905562 Zbl 1254.16020

[Vancliff and Van Rompay 2000] M. Vancliff and K. Van Rompay, "Four-dimensional regular algebras with point scheme, a nonsingular quadric in $\mathbf{P}^{3}$ ", Comm. Algebra 28:5 (2000), 2211-2242. MR 1757458 Zbl 0961.16019

[Vancliff et al. 1998] M. Vancliff, K. Van Rompay, and L. Willaert, "Some quantum $\mathbf{P}^{3}$ s with finitely many points”, Comm. Algebra 26:4 (1998), 1193-1208. MR 1612220 Zbl 0915.16035

Communicated by Efim Zelmanov

Received 2015-04-07 Revised 2016-02-07 Accepted 2016-03-10

kenhchan@math.washington.edu

Department of Mathematics, University of Washington, Box 354350, Seattle, WA 98195-4350, United States

ayoung@digipen.edu

Department of Mathematics, DigiPen Institute of Technology, 9931 Willows Road NE, Redmond, WA 98052, United States

zhang@math.washington.edu

Department of Mathematics, University of Washington, Box 354350, Seattle, WA 98195-4350, United States 


\section{Algebra \& Number Theory}

msp.org/ant

\section{EDITORS}

MANAGING EDITOR

Bjorn Poonen

Massachusetts Institute of Technology

Cambridge, USA

\author{
EDITORIAL BOARD CHAIR \\ David Eisenbud \\ University of California \\ Berkeley, USA
}

\section{BOARD OF EDITORS}

Georgia Benkart

Dave Benson

Richard E. Borcherds

John H. Coates

J-L. Colliot-Thélène

Brian D. Conrad

Hélène Esnault

Hubert Flenner

Sergey Fomin

Edward Frenkel

Andrew Granville

Joseph Gubeladze

Roger Heath-Brown

Craig Huneke

Kiran S. Kedlaya

János Kollár

Yuri Manin

Philippe Michel
University of Wisconsin, Madison, USA

University of Aberdeen, Scotland

University of California, Berkeley, USA

University of Cambridge, UK

CNRS, Université Paris-Sud, France

Stanford University, USA

Freie Universität Berlin, Germany

Ruhr-Universität, Germany

University of Michigan, USA

University of California, Berkeley, USA

Université de Montréal, Canada

San Francisco State University, USA

Oxford University, UK

University of Virginia, USA

Univ. of California, San Diego, USA

Princeton University, USA

Northwestern University, USA

École Polytechnique Fédérale de Lausanne
Susan Montgomery

Shigefumi Mori

Raman Parimala

Jonathan Pila

Anand Pillay

Victor Reiner

Peter Sarnak

Joseph H. Silverman

Michael Singer

Vasudevan Srinivas

J. Toby Stafford

Ravi Vakil

Michel van den Bergh

Marie-France Vignéras

Kei-Ichi Watanabe

Efim Zelmanov

Shou-Wu Zhang
University of Southern California, USA

RIMS, Kyoto University, Japan

Emory University, USA

University of Oxford, UK

University of Notre Dame, USA

University of Minnesota, USA

Princeton University, USA

Brown University, USA

North Carolina State University, USA

Tata Inst. of Fund. Research, India

University of Michigan, USA

Stanford University, USA

Hasselt University, Belgium

Université Paris VII, France

Nihon University, Japan

University of California, San Diego, USA

Princeton University, USA

\section{PRODUCTION}

production@msp.org

Silvio Levy, Scientific Editor

See inside back cover or msp.org/ant for submission instructions.

The subscription price for 2016 is US \$/year for the electronic version, and \$/year (+\$, if shipping outside the US) for print and electronic. Subscriptions, requests for back issues and changes of subscribers address should be sent to MSP.

Algebra \& Number Theory (ISSN 1944-7833 electronic, 1937-0652 printed) at Mathematical Sciences Publishers, 798 Evans Hall \#3840, c/o University of California, Berkeley, CA 94720-3840 is published continuously online. Periodical rate postage paid at Berkeley, CA 94704, and additional mailing offices.

ANT peer review and production are managed by EditFLOW ${ }^{\circledR}$ from MSP.

\section{PUBLISHED BY}

mathematical sciences publishers

nonprofit scientific publishing

http://msp.org/

(C) 2016 Mathematical Sciences Publishers 


\section{Algebra \& Number Theory}

Volume $10 \quad$ No. $3 \quad 2016$

Group schemes and local densities of ramified hermitian lattices in residue characteristic 2: Part I

SUNGMUN CHO

Presentation of affine Kac-Moody groups over rings

DANIEL ALLCOCK

Discriminant formulas and applications

Kenneth Chan, Alexander A. Young and James J. Zhang

LUIS E. GARCIA

Multiple period integrals and cohomology

RoElof W. BRUGGEMAN and YoungJu CHOIE

The existential theory of equicharacteristic henselian valued fields

SYLVY ANSCOMBE and ARNO FEHM

SOMNATH JHA, TADASHI OCHIAI and GERGELY ZÁBRÁDI 